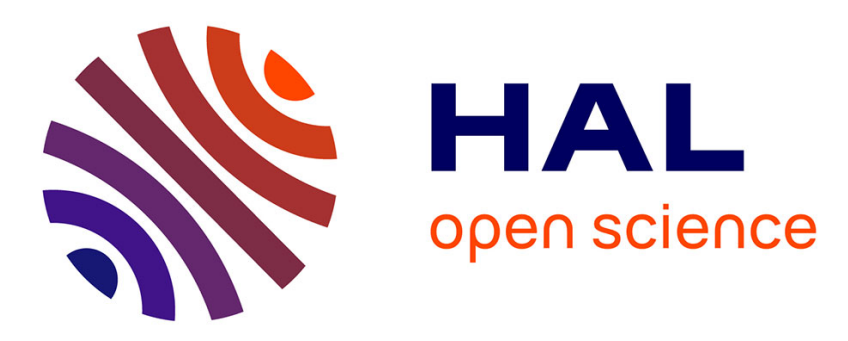

\title{
Geometric Numerical Methods and Results in the Control Imaging Problem in Nuclear Magnetic Resonance
}

\author{
Bernard Bonnard, Olivier Cots
}

\section{To cite this version:}

Bernard Bonnard, Olivier Cots. Geometric Numerical Methods and Results in the Control Imaging Problem in Nuclear Magnetic Resonance. Mathematical Models and Methods in Applied Sciences, 2014, vol. 24 ( $\mathrm{n}^{\circ}$ 1), pp. 187-212. 10.1142/S0218202513500504 . hal-01136896

\section{HAL Id: hal-01136896 https://hal.science/hal-01136896}

Submitted on 30 Mar 2015

HAL is a multi-disciplinary open access archive for the deposit and dissemination of scientific research documents, whether they are published or not. The documents may come from teaching and research institutions in France or abroad, or from public or private research centers.
L'archive ouverte pluridisciplinaire HAL, est destinée au dépôt et à la diffusion de documents scientifiques de niveau recherche, publiés ou non, émanant des établissements d'enseignement et de recherche français ou étrangers, des laboratoires publics ou privés. 


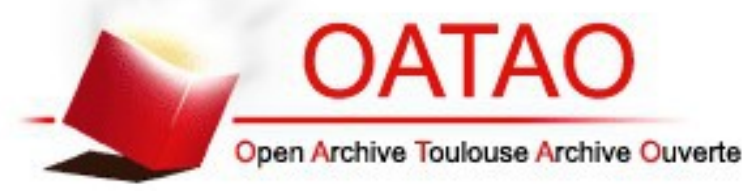

\section{Open Archive TOULOUSE Archive Ouverte (OATAO)}

OATAO is an open access repository that collects the work of Toulouse researchers and makes it freely available over the web where possible.

This is an author-deposited version published in : http://oatao.univ-toulouse.fr/ Eprints ID : 12317

To link to this article : DOI :10.1142/S0218202513500504

URL : http://dx.doi.org/10.1142/S0218202513500504

To cite this version : Bonnard, Bernard and Cots, Olivier Geometric Numerical Methods and Results in the Control Imaging Problem in Nuclear Magnetic Resonance. (2014) Mathematical Models and Methods in Applied Sciences, vol. 24 ( $\left.n^{\circ} 1\right)$. pp. 187-212. ISSN 02182025

Any correspondance concerning this service should be sent to the repository administrator: staff-oatao@,listes-diff.inp-toulouse.fr 


\title{
GEOMETRIC NUMERICAL METHODS AND RESULTS IN THE CONTRAST IMAGING PROBLEM IN NUCLEAR MAGNETIC RESONANCE
}

\author{
B. BONNARD* and O. COTS $^{\dagger}$ \\ Institut de Mathématiques de Bourgogne, \\ UMR CNRS 5584, 9 Avenue Alain Savary, \\ BP 47870 F-21078 DIJON Cedex France \\ *bernard.bonnard@u-bourgogne.fr \\ †olivier.cots@u-bourgogne.fr
}

\begin{abstract}
The purpose of this paper is to present numerical methods and results about the contrast imaging problem in nuclear magnetic resonance which corresponds to a Mayer problem in optimal control. The candidates as minimizers are selected among a set of extremals, solutions of a Hamiltonian system given by the Pontryagin Maximum Principle and sufficient second order conditions are described. They form the geometric foundations of the HАмРАтн code which combines shooting and continuation methods, see Ref. 9. The main contribution of this paper is to present a numerical analysis of the contrast imaging problem in NMR in the case of deoxygenated/oxygenated blood samples as an application of the aforementioned techniques.
\end{abstract}

Keywords: Geometric optimal control; contrast imaging in NMR; shooting and continuation methods.

\section{Introduction}

Geometric optimal control was introduced very recently in nuclear magnetic resonance (NMR) to control the dynamics of spin particles, e.g. see Ref. 7. The model is the coupling of a system equations introduced by F. Bloch in 1946, describing 
the motion of nuclear magnetization:

$$
\begin{aligned}
& \frac{\mathrm{d} M_{x}}{\mathrm{~d} t}=-\frac{M_{x}}{T_{2}}-\Delta \omega M_{y}+\omega_{y} M_{z}, \\
& \frac{\mathrm{d} M_{y}}{\mathrm{~d} t}=-\frac{M_{y}}{T_{2}}+\Delta \omega M_{x}-\omega_{x} M_{z}, \\
& \frac{\mathrm{d} M_{z}}{\mathrm{~d} t}=\frac{\left(M_{0}-M_{z}\right)}{T_{1}}-\omega_{y} M_{x}+\omega_{x} M_{y},
\end{aligned}
$$

where $M=\left(M_{x}, M_{y}, M_{z}\right)$ is the magnetization vector, $M_{0}$ represents the equilibrium state of the system, $T_{1}, T_{2}$ are the relaxation times characteristic of the particle, $\Delta w$ is the detuning and $\omega=\left(\omega_{x}, \omega_{y}\right)$ is the control which is a transverse radio frequency field in the $(x, y)$-plane. This model represents the physical experiment in NMR spectroscopy very accurately and the control can be realized in practice as shown, for instance, by the saturation problem of a spin 1/2 particle which consists in bringing the magnetization vector to zero, see Ref. 15 .

An ideal model in the contrast imaging problem in NMR consists of considering a system of two Bloch Eq. (1.1) with zero detuning and distinct relaxation parameters representing two different spin $1 / 2$ particles, controlled by the same magnetic field and bringing the magnetization of the first particle to zero while maximizing the magnitude of the magnetization vector of the second particle.

This is illustrated by the experimental results, presented on Fig. 1. The sample consists of two test tubes with outer diameters of $5 \mathrm{~mm}$ and $8 \mathrm{~mm}$. The outer and inner volumes were respectively filled with two solutions of oxygenated and deoxygenated blood. The left-hand side represents the two samples at the equilibrium (they both appear white) and the right-hand side shows the result after the
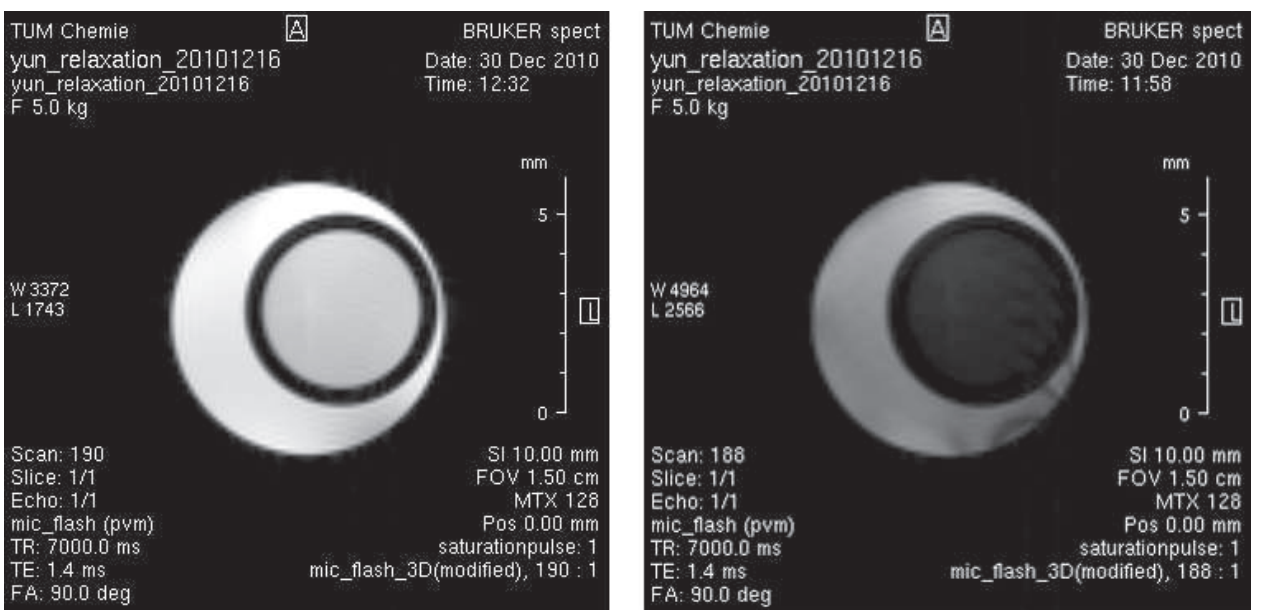

Fig. 1. Experimental results: The inner circle shape sample mimics the deoxygenated blood, where $T_{1}=1.3 \mathrm{~s}$ and $T_{2}=50 \mathrm{~ms}$; the outside moon shape sample corresponds to the oxygenated blood, where $T_{1}=1.3 \mathrm{~s}$ and $T_{2}=200 \mathrm{~ms}$. 
application of the control sequence. The inner sample appears black (zero magnetization) while the outer sample represents the remaining magnetization. For details, see Refs. 4 and 16.

In particular, using the symmetry of revolution of each spin system with respect to the $z$-axis, we shall concentrate on the case where $\omega_{y} \equiv 0$ and each spin system can be restricted to the $(y, z)$-plane, defining the system:

$$
\begin{aligned}
& \frac{\mathrm{d} M_{y}^{i}}{\mathrm{~d} t}=-\frac{M_{y}^{i}}{T_{2}^{i}}-\omega_{x} M_{z}^{i}, \\
& \frac{\mathrm{d} M_{z}^{i}}{\mathrm{~d} t}=\frac{\left(M_{0}^{i}-M_{z}^{i}\right)}{T_{1}^{i}}+\omega_{x} M_{y}^{i}, \quad i=1,2 .
\end{aligned}
$$

The contrast problem is then the following optimal control problem: transfer the system from the equilibrium state in a given transfer time $t_{f}$ to $M^{1}\left(t_{f}\right)=0$ while maximizing $\left|M^{2}\left(t_{f}\right)\right|^{2}$ and $\left|M^{2}\left(t_{f}\right)\right|$ represents the contrast.

This problem corresponds to a Mayer problem in optimal control:

$$
\min _{u(\cdot)} c\left(x\left(t_{f}\right)\right), \quad \frac{\mathrm{d} x}{\mathrm{~d} t}=f(x, u), \quad u \in U,
$$

with the boundary conditions:

$$
x(0)=x_{0}, \quad g\left(x\left(t_{f}\right)\right)=0
$$

and it can be analyzed using Pontryagin Maximum Principle, see Ref. 2. It tells us that optimal solutions are found as projections on the $x$-space as extremals solutions of the Hamiltonian system:

$$
\begin{aligned}
& \frac{\mathrm{d} x}{\mathrm{~d} t}=\frac{\partial H}{\partial p}(x, p, u), \quad \frac{\mathrm{d} p}{\mathrm{~d} t}=-\frac{\partial H}{\partial x}(x, p, u), \\
& H(x, p, u)=\max _{v \in U} H(x, p, v),
\end{aligned}
$$

where $H(x, p, u)=\langle p, f(x, u)\rangle$ is the pseudo-Hamiltonian and $p(\cdot)$ is the adjoint vector. Moreover the solution has to satisfy the transversality condition:

$$
p\left(t_{f}\right)=p^{0} \frac{\partial c}{\partial x}\left(x\left(t_{f}\right)\right)+\sum_{i=1}^{k} \sigma_{i} \frac{\partial g_{i}}{\partial x}\left(x\left(t_{f}\right)\right), \quad p^{0} \leq 0 .
$$

The Maximum Principle is only a necessarily optimality condition and it was recently completed by generalizing the concept of conjugate point and extremal field in standard calculus of variation, see Ref. 12, to get sufficient geometric optimality conditions, see Ref. 5.

All this theoretical framework will be rapidly recalled in this paper before presenting the main contribution of this paper consisting in describing the algorithms and simulations in one specific example in the contrast imaging problem. The general method which is the core of the HAMPATH code, Ref. 10, is the following. 
The Maximum Principle together with the transversality condition leads to the computation of the optimal solution using a shooting equation: $S(p(0))=0$, integrating the ODE according to Eq. (1.3) and solving with prescribed boundary conditions to compute an initial condition on the adjoint vector $p(0)$. If the method is simple, an initial guess has to be determined to ensure the convergence of the shooting. Hence the shooting is complemented by a smooth continuation method. The Hamiltonian dynamics defined by Eq. (1.3) is embedded into a one-parameter family of Hamiltonian dynamics $H_{\lambda}, \lambda \in[0,1]$ and this leads to a one-parameter family of shooting equations: $S(p(0), \lambda)=0$. The connected component of the set of zeros $p_{\lambda}(0)$ will form a differential curve which is followed from $\lambda=0$ to $\lambda=1$ to compute the solution. Also the differential of this curve is shown to be related to the concept of conjugate point.

In order to be applied to the contrast imaging problem one must adapt this general algorithm. Indeed in the general case of Eq. (1.1) due to the symmetry of revolution there is a one-parameter family of solutions of the shooting equation. One adaptation concerning the case treated in this paper restricting the control to $\omega_{y} \equiv 0$ is the following. This case leads to a single-input control system of the form:

$$
\frac{\mathrm{d} x}{\mathrm{~d} t}=F(x)+u G(x), \quad|u| \leq 1 .
$$

In the single-input case, the optimal solution is a concatenation of regular arcs where the control is given by $u(t)=\operatorname{sgn}\langle p(t), G(x(t))\rangle$ and singular arcs where $\langle p(t), G(x(t))\rangle=0$, identically. An optimal solution is a concatenation of bang arcs with $u= \pm 1$ and singular arcs and the problem is to determine the sequence of such arcs depending upon the transfer time and the relaxation parameters.

For that the technique is to use a regularization of the optimal control problem transforming the Mayer problem into a Bolza problem, for instance with cost:

$$
c\left(x\left(t_{f}\right)\right)+(1-\lambda) \int_{0}^{t_{f}}|u|^{2-\lambda} \mathrm{d} t, \quad \lambda \in[0,1] .
$$

Once the structure of the solution is known, it is computed using a multiple-shooting method. In particular, in the case of a deoxygenated/oxygenated blood samples, one proves in this paper that the optimal solution is the concatenation of a bang and a singular arc which is finally accurately computed.

This paper is organized as Follows: In Sec. 2, we present in details the model used in the contrast imaging problem. In Sec. 3, we recall the necessary and sufficient optimality conditions with applications to our problem. The main results in this paper are finally presented in Secs. 4 and 5, which respectively describe the algorithmic schemes and the numerical simulations in the study case of deoxygenated/oxygenated blood samples. 


\section{The Mathematical Model and the Mayer Optimal Control Problem}

According to Eq. (1.2) using normalized coordinates, one considers the system formed by coupling two spin $1 / 2$ systems with zero detuning, described for $i=1,2$ by:

$$
\begin{aligned}
& \frac{\mathrm{d} y_{i}}{\mathrm{~d} t}=-\Gamma_{i} y_{i}-u z_{i}, \\
& \frac{\mathrm{d} z_{i}}{\mathrm{~d} t}=\gamma_{i}\left(1-z_{i}\right)+u y_{i},
\end{aligned}
$$

with $\Gamma_{i}=2 \pi /\left(\omega_{\max } T_{2}^{i}\right), \gamma_{i}=2 \pi /\left(\omega_{\max } T_{1}^{i}\right)$ while the control is bounded by $|u| \leq 2 \pi$. From the experimental point of view, $\omega_{\max } / 2 \pi$ can be chosen up to $15,000 \mathrm{~Hz}$ but the value $32.3 \mathrm{~Hz}$ will be considered in our experiments, see Ref. 15 for more details. For the experiments one considers four cases, the relaxation times being:

(1) Cerebrospinal fluid

$: T_{1}=2000 \mathrm{~ms}, \quad T_{2}=200 \mathrm{~ms}$

Water

$: T_{1}=2500 \mathrm{~ms}, \quad T_{2}=2500 \mathrm{~ms}$

(2) Deoxygenated blood

$: T_{1}=1350 \mathrm{~ms}, \quad T_{2}=50 \mathrm{~ms}$

Oxygenated blood

$: T_{1}=1350 \mathrm{~ms}, \quad T_{2}=200 \mathrm{~ms}$

(3) Gray matter of cerebrum : $T_{1}=920 \mathrm{~ms}, \quad T_{2}=100 \mathrm{~ms}$

White matter of cerebrum : $T_{1}=780 \mathrm{~ms}, \quad T_{2}=90 \mathrm{~ms}$

(4) Water

$: T_{1}=2500 \mathrm{~ms}, \quad T_{2}=2500 \mathrm{~ms}$

Fat

$: T_{1}=200 \mathrm{~ms}, \quad T_{2}=100 \mathrm{~ms}$

Denoting $x_{1}=\left(y_{1}, z_{1}\right), x_{2}=\left(y_{2}, z_{2}\right)$ as the vectors representing each spin, the Bloch ball $\left|x_{i}\right| \leq 1$ being invariant for the dynamics since the parameters satisfy $2 \Gamma_{i} \geq \gamma_{i}$, the optimal control problem is the following: starting from the north pole $N=((0,1),(0,1))$ corresponding to the equilibrium point of the uncontrolled dynamics, the goal is to reach in a given transfer time $t_{f}$ the final state $x_{1}\left(t_{f}\right)=0$ corresponding to zero magnetization of the first spin, while maximizing the square norm $\left|x_{2}\left(t_{f}\right)\right|^{2}$ of the second spin.

Introducing the system $\dot{x}=f(x, u)$, where $x=\left(x_{1}, x_{2}\right)$ belongs to $\left|x_{i}\right| \leq 1$ and $|u| \leq 2 \pi$, formed by the coupling of two equations Eq. (2.1), with $x(0)=N$, $g\left(x\left(t_{f}\right)\right)=x_{1}\left(t_{f}\right)$ and with the cost functions $c\left(x\left(t_{f}\right)\right)=-\left|x_{2}\left(t_{f}\right)\right|^{2}$, the problem is a standard Mayer problem given by:

- a system $\dot{x}=f(x, u), u \in U, x(0)=x_{0}$, where $U$ is the control domain and $\mathcal{U}$ is the set of admissible controls,

- a final boundary condition $g\left(x\left(t_{f}\right)\right)=0$, and

- a cost to minimize, $\min _{u(\cdot) \in \mathcal{U}} c\left(x\left(t_{f}\right)\right)$. 


\section{Necessary and Sufficient Optimality Conditions for a Mayer Problem}

\subsection{Notation}

Let $X$ be an open manifold of $\mathbb{R}^{n}, T_{x} X, T_{x}^{*} X$ the tangent and cotangent spaces at $x \in X$. If $F, G$ are two smooth vector fields, the Lie bracket is given by:

$$
[F, G](x)=\frac{\partial F}{\partial x}(x) G(x)-\frac{\partial G}{\partial x}(x) F(x),
$$

while the Poisson bracket of two smooth Hamiltonians $H_{1}, H_{2}$ (the associated Hamiltonian vector fields being denoted $\left.\mathbf{H}_{i}\right)$ is defined by $\left\{H_{1}, H_{2}\right\}(z)=\mathrm{d} H_{1}\left(\mathbf{H}_{2}\right)$, $z=(x, p)$. Denoting $H_{F}, H_{G}$ the Hamiltonian lifts of $F, G: H_{F}=\langle p, F(x)\rangle$, $H_{G}=\langle p, G(x)\rangle$, one has the relation $\left\{H_{F}, H_{G}\right\}=\langle p,[F, G](x)\rangle$.

\subsection{Geometric preliminaries}

To highlight the necessary optimality conditions, one introduces the following geometric framework. Consider a (smooth) Mayer problem of the form:

$$
\min _{u(\cdot) \in \mathcal{U}} c\left(x\left(t_{f}\right)\right), \quad \frac{\mathrm{d} x}{\mathrm{~d} t}=F(x)+u G(x),
$$

$x \in X \subset \mathbb{R}^{n},|u| \leq 1$, with fixed initial condition: $x(0)=x_{0}$ with the terminal condition $x\left(t_{f}\right) \in M$, where $M$ is a submanifold of codimension $k$ of $X$ defined by: $g(x)=0$. The class of admissible controls $\mathcal{U}$ is the set of $L^{\infty}$-mappings, valued in $U:|u| \leq 1$ such that the solution $x\left(., x_{0}, u\right)$ is defined on $\left[0, t_{f}\right]$. For $t \leq t_{f}$ one defines the accessibility set at time $t: A\left(x_{0}, t\right)=\bigcup_{u(\cdot) \in \mathcal{U}} x\left(t, x_{0}, u\right)$. Assuming that the cost $c$ is a regular mapping, such that for $c(x)=m$, the set $M_{m}=\{x ; g(x)=0, c(x)=m\}$ is a submanifold of codimension $k+1$, one gets the following necessary optimality condition according to the Pontryagin Maximum Principle and its extension, see Refs. 2 and 13.

\subsection{Maximum principle and high order maximum principle}

Theorem 3.1. Let $u^{*}(\cdot)$ be an admissible control whose corresponding trajectory $x^{*}(\cdot)$ is optimal on $\left[0, t_{f}\right]$. Then there exists a nonzero adjoint vector function $p^{*}(\cdot)$ such that the conditions

$$
\frac{\mathrm{d} x^{*}}{\mathrm{~d} t}=\frac{\partial H}{\partial p}\left(x^{*}, p^{*}, u^{*}\right), \quad \frac{\mathrm{d} p^{*}}{\mathrm{~d} t}=-\frac{\partial H}{\partial x}\left(x^{*}, p^{*}, u^{*}\right)
$$

are satisfied almost everywhere, where $H=\langle p, F+u G\rangle$ is the pseudo-Hamiltonian. Denoting $M(x, p)=\max _{|u| \leq 1} H(x, p, u)$ one must have a.e. the maximization 
condition

$$
H\left(x^{*}, p^{*}, u^{*}\right)=M\left(x^{*}, p^{*}\right)
$$

and moreover $M$ is a constant.

In the singular case where $H_{G}=0$ and if the control is not saturating, i.e. $\left|u^{*}\right|<1$, the generalized Legendre-Clebsch condition,

$$
\left\{H_{G},\left\{H_{G}, H_{F}\right\}\right\} \leq 0
$$

has to be satisfied.

Finally we have the boundary conditions

$$
g\left(x^{*}\left(t_{f}\right)\right)=0
$$

and

$$
p^{*}\left(t_{f}\right)=p_{0} \frac{\partial c}{\partial x}\left(x^{*}\left(t_{f}\right)\right)+\sum_{i=1}^{k} \sigma_{i} \frac{\partial g_{i}}{\partial x}\left(x^{*}\left(t_{f}\right)\right),
$$

$p_{0} \leq 0, \sigma=\left(\sigma_{1}, \ldots, \sigma_{k}\right) \in \mathbb{R}^{k}$. This last condition is called the transversality condition.

Definition 3.1. We call any solution of Eqs. (3.1) and (3.2) an extremal, and if the boundary conditions Eqs. (3.4), (3.5) are satisfied they are called BCextremals. Condition (3.2) imposes $u(t)=\operatorname{sgn}\langle p(t), G(x(t))\rangle$ at any time such that $\langle p(t), G(x(t))\rangle \neq 0$. An extremal is called regular if $u(t)=\operatorname{sgn} H_{G}$ and bang-bang if the number of switchings is finite. It is called singular if $H_{G}=0$.

\subsection{Computation of singular extremals (Ref. 3)}

This computation is standard: let $z(\cdot)=(x(\cdot), p(\cdot))$ be an extremal, $t \in\left[0, t_{f}\right]$. In the singular case, we have $H_{G}(z(t))=0$ identically. Differentiating with respect to time,

$$
\begin{gathered}
H_{G}(z(t))=\left\{H_{G}, H_{F}\right\}(z(t))=0 \\
\left\{\left\{H_{G}, H_{F}\right\}, H_{F}\right\}(z(t))+u_{s}(t)\left\{\left\{H_{G}, H_{F}\right\}, H_{G}\right\}(z(t))=0
\end{gathered}
$$

and Eq. (3.7) defines the singular control $u_{s}$ as a dynamic feedback, outside $S=\left\{z ;\left\{\left\{H_{G}, H_{F}\right\}, H_{G}\right\}(z)=0\right\}$. Denote $\Sigma$ the switching surface $H_{G}=0$ and $\Sigma^{\prime}: H_{G}=\left\{H_{G}, H_{F}\right\}=0$. Substituting $u_{s}$ into $H$ defines a true Hamiltonian function denoted $H_{s}$ outside $S$. We have:

Proposition 3.1. Outside $S$, the singular extremals are the solutions of

$$
\frac{\mathrm{d} x}{\mathrm{~d} t}=\frac{\partial H_{s}}{\partial p}, \quad \frac{\mathrm{d} p}{\mathrm{~d} t}=-\frac{\partial H_{s}}{\partial x},
$$

starting at $t=0$ from $\Sigma^{\prime} \backslash S$ and they are contained in $\Sigma^{\prime}$. 
For the computations of the singular trajectories, we need the following Lie brackets:

$$
\begin{aligned}
F & =\sum_{i=1,2}\left(-\Gamma_{i} y_{i}\right) \frac{\partial}{\partial y_{i}}+\left(\gamma_{i}\left(1-z_{i}\right)\right) \frac{\partial}{\partial z_{i}}, \\
G & =\sum_{i=1,2}-z_{i} \frac{\partial}{\partial y_{i}}+y_{i} \frac{\partial}{\partial z_{i}}, \\
{[G, F] } & =\sum_{i=1,2}\left(\gamma_{i}-\delta_{i} z_{i}\right) \frac{\partial}{\partial y_{i}}-\left(\delta_{i} y_{i}\right) \frac{\partial}{\partial z_{i}}, \\
{[[G, F], F] } & =\sum_{i=1,2}\left(\gamma_{i}\left(\gamma_{i}-2 \Gamma_{i}\right)-\delta_{i}^{2} z_{i}\right) \frac{\partial}{\partial y_{i}}+\delta_{i}^{2} y_{i} \frac{\partial}{\partial z_{i}}, \\
{[[G, F], G] } & =\sum_{i=1,2} 2 \delta_{i} y_{i} \frac{\partial}{\partial y_{i}}+\left(\gamma_{i}-2 \delta_{i} z_{i}\right) \frac{\partial}{\partial z_{i}},
\end{aligned}
$$

where $\delta_{i}=\gamma_{i}-\Gamma_{i}$.

\subsection{Application to the contrast problem: Boundary conditions}

Since the Bloch ball is invariant for the dynamics, one can take $X=\left\{\left|x_{i}\right|<1 ; i=\right.$ 1,2\}. Applying the Maximum Principle, the boundary conditions Eqs. (3.4) and (3.5) give us:

$$
\begin{aligned}
& x_{1}\left(t_{f}\right)=0 \quad \text { (zero magnetization of the first spin) } \\
& p_{2}\left(t_{f}\right)=-2 p^{0} x_{2}\left(t_{f}\right), \quad p^{0} \leq 0 .
\end{aligned}
$$

If $p^{0}$ is nonzero, it can be normalized by homogeneity to $p^{0}=-1 / 2$. The case $p^{0}=0$ corresponds to the case where $t_{f}$ is the minimum time to transfer the first spin to zero.

\subsection{Second-order optimality conditions}

This section relies on the work of Ref. 5 .

Definition 3.2. Let $z(\cdot)$ be a reference singular solution of $\mathbf{H}_{s}$ on $\left[0, t_{f}\right]$ and contained in $\Sigma^{\prime}$. The variational equation

$$
\begin{gathered}
\delta \dot{z}=\mathrm{d}_{s}(z(t)) \delta z, \\
\mathrm{~d} H_{G}=\mathrm{d}\left\{H_{F}, H_{G}\right\}=0
\end{gathered}
$$

is called Jacobi equation. A Jacobi field $J(t)=(\delta x, \delta p)$ is a nonzero solution of the Jacobi equation. It is said semi-vertical at time $t$ if $\delta x(t) \in \mathbb{R} G(x(t))$. The times $0 \leq t_{1}<t_{2} \leq t_{f}$ are said to be conjugate if there exists a Jacobi field $J(t)$ semi-vertical at $t=t_{1}, t_{2}$ and the points $x_{1}=x\left(t_{1}\right)$ and $x_{2}=x\left(t_{2}\right)$ are said to be conjugate. 
Assumptions. Let $z(\cdot)=(x(\cdot), p(\cdot))$ be a reference singular extremal curve on $\left[0, t_{f}\right]$ solution on $\Sigma^{\prime} \backslash S$ and we assume the following:

(A1) $t \mapsto x(t)$ is a one-to-one immersion. Applying a feedback, one can identify the reference singular control to zero.

(A2) Strong corank one condition.

$K(t)=\operatorname{span}\left\{\operatorname{ad}^{k} F \cdot G(x(t)) ; k \geq 0, t \in\left[0, t_{f}\right]\right\}$ is of codimension one and generated by the first $(n-1)$ vectors $\left\{\operatorname{ad}^{k} F \cdot G(x(t)) ; k=0, \ldots, n-2\right\}$ where ad $F \cdot G$ denotes $[F, G]$.

(A3) Non-exceptional case. $H_{s}(z(t))$ is nonzero.

\subsubsection{Optimality results}

One considers a Mayer problem for a system $\dot{x}=F(x)+u G(x), x \in X$ open set of $\mathbb{R}^{n},|u| \leq 2 \pi, \min _{u(\cdot)} c\left(x\left(t_{f}\right)\right)$ and one assumes that the terminal manifold $M$ is a trajectory of the vector field $G:\{\exp t G ; t \in \mathbb{R}\}$. In this case one will derive a sufficient optimality condition, which is applicable to the contrast imaging problem.

First one recalls the following proposition from Ref. 5 .

Proposition 3.2. Assuming $u \in \mathbb{R}$, a necessary optimality condition in the Mayer problem for a singular arc $\delta_{s}$ on $\left[0, t_{f}\right]$ satisfying $(\mathrm{A} 1),(\mathrm{A} 2)$ and $(\mathrm{A} 3)$ is the nonexistence of a time $0<t_{c}<t_{f}$ conjugate to zero, for the solutions of the system contained in a tubular neighborhood of $\delta_{s}$.

Proof. According to Ref. 5, the first conjugate time $0<t_{1 c}$ is the first time $t$ such that the extremity mapping $E^{x_{1}, t}: u(\cdot) \mapsto x\left(t, x_{1}, u\right)$ becomes open when restricting to curves contained in a tubular neighborhood of $\delta_{s}$. This is clearly a necessary optimality condition.

Next, one presents the following sufficient optimality condition.

Theorem 3.2. Consider the Mayer problem with the terminal manifold $M=\{\exp t G ; t \in \mathbb{R}\}$. Let $\delta_{+} \delta_{s}$ be a BC-extremal on $\left[0, t_{f}\right]$ formed by a bang arc with $u=2 \pi$ on $\left[0, t_{1}\right]$ followed by a singular arc on $\left[t_{1}, t_{f}\right]$. Assume that the singular arc $t \mapsto \delta_{s}(t)$ satisfies the assumptions (A1), (A2), (A3) and is associated to a control $u_{s}(\cdot)$ on $\left[t_{1}, t_{f}\right]$ which is not saturating, i.e. $\left|u_{s}\right|<2 \pi$. Then the extremal $\delta_{+} \delta_{s}$ is optimal on $\left[0, t_{f}\right]$ in a tubular neighborhood of $\delta_{s}$ provided there exists no conjugate point on $\left[t_{1}, t_{f}\right]$.

Proof. (We use Ref. 19) Denote $x_{f}$ the terminal point $\delta_{s}\left(t_{f}\right)$ and $p_{f}$ the adjoint vector. One has $\left\langle p_{f}, G\left(x_{f}\right)\right\rangle=0$. According to the classification of extremals due to Ref. 14, near $\left(x_{f}, p_{f}\right)$ every extremal is of the form $\delta_{ \pm} \delta_{s} \delta_{ \pm}$, where an arc of the sequence can be empty. Let $M_{\varepsilon}=\left\{\exp s G\left(x_{f}\right) ;|s|<\varepsilon\right.$ small enough $\}$. Using the transversality condition, near $\left(x_{f}, p_{f}\right)$ every BC-extremal starting from $M_{\varepsilon}$ is of the form $\delta_{ \pm} \delta_{s}$. 
Let $S\left(x_{f}, t\right), t \in\left[t_{1}, t_{f}\right]$ be the surface formed by integrating backwards in time the singular flow initiating from $M_{\varepsilon}$. For fixed $t$, it is a surface of codimension two, whose tangent space can be easily computed at a point $\delta_{s}(t), t \in\left[t_{1}, t_{f}\right]$. It is a plane $E(t)$ spanned by $\mathrm{d} \Pi(J(t)), J(t)$ being the Jacobi fields which are semi-vertical at $t_{f}$, where $\Pi$ is the standard projection: $(x, p) \mapsto x$.

Hence if $\operatorname{rank}\{E, F, G\}_{\mid \delta_{s}(t)}$ is four for $t<t_{f}$, then a policy $\delta_{ \pm} \delta_{s}$ will form an extremal field along the reference singular extremal $\delta_{s}$. Otherwise, if it is not of full rank, at a time $t$, then by definition $\delta_{s}(t)$ and $\delta_{s}\left(t_{f}\right)$ are conjugate. Now clearly, since conjugate times correspond to points where the extremity mapping becomes open, if $\delta_{s}(t)$ and $\delta_{s}\left(t_{f}\right)$ are conjugate for $t_{1} \leq t<t_{f}$, then there exists a pair of conjugate points on $\left[t_{1}, t_{f}\right]$ and a point conjugate to $x\left(t_{1}\right)$.

\subsubsection{Application to the contrast problem}

First of all in the contrast problem where the control bound is normalized to $2 \pi$, one has $\Gamma_{i}, \gamma_{i} \ll 1$. Hence a bang solution amounts roughly to a rotation of each plane $\left(y_{i}, z_{i}\right)$ around 0 . Moreover, the north pole $N=((0,1),(0,1))$, which is the initial state of the Mayer problem, is an equilibrium point for the singular control system and due to symmetry of revolution, one can take for the first bang either $u=+2 \pi$ or $u=-2 \pi$. At the final state the first spin has to be at zero, which is the center of the rotation of the first spin. Hence the terminal manifold is indeed a trajectory of the vector field $G$. In particular, one deduces:

Lemma 3.1. The simplest BC-extremal in the contrast problem is of the form $\delta_{+} \delta_{s}$.

3.6.3. The limit case $t_{f}=t_{\mathrm{min}}$, minimum time to transfer the first spin to zero

An interesting situation studied in Ref. 15 is the limit case when the transfer time $t_{f}$ is exactly the minimum time $t_{\text {min }}$ to transfer the first spin to zero. We recall the optimal policy in the physical interesting situation.

Proposition 3.3. Assume $2 \Gamma_{1}>3 \gamma_{1}$, then the time minimal solution to transfer the first spin from the north pole to the center of Bloch ball is of the form $\delta_{+} \delta_{s} \delta_{+} \delta_{s}$ and contains two non-empty singular arcs, the first one being horizontal in the plane $\left(y_{1}, z_{1}\right)$ while the second is a subarc of the vertical axis of revolution $O z_{1}$.

It shows that in the physical application if the transfer time is $t_{\min }$, then the policy $\delta_{+} \delta_{s}$ is not optimal. Also it gives in each experiment an indication about the relation between the contrast and the relaxation parameters. The corresponding contrast is called the Sat-contrast, see Ref. 6. We represent in Fig. 2, the time minimal synthesis to steer the system from the north pole to any point of the Bloch ball. In particular, it shows the time minimal solution of the saturation problem. 


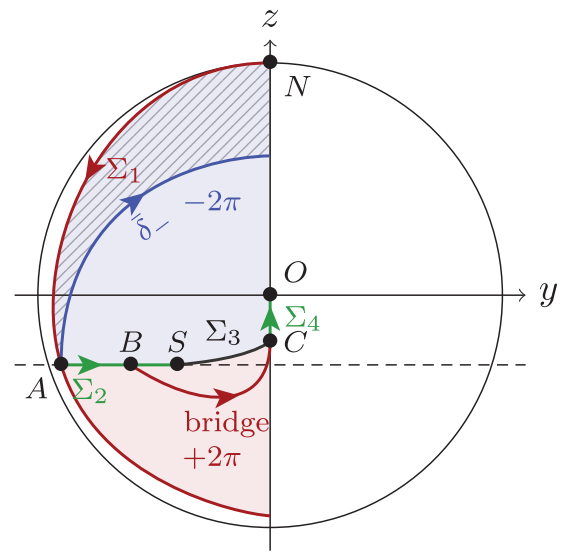

Fig. 2. (Color online) Schematic time minimal synthesis to steer a single spin system from the north pole $\mathrm{N}$ to any point of the Bloch ball. An arbitrary zoom has been used to construct the figure. The set of $\Sigma_{i}$ forms the switching surface $\Sigma$ dividing the $+2 \pi$ and $-2 \pi$ areas respectively in red and blue. The $\bar{\delta}_{-}$bang arc splits two domains. The hatched one has bang-bang solutions whereas the optimal solutions of the other one contain at least one nontrivial singular arc. The minimal time trajectory to steer the spin from $\mathrm{N}$ to $\mathrm{O}$ (the center of the Bloch ball) is $N A B C O$, i.e. it is of the form $\delta_{+} \delta_{s} \delta_{+} \delta_{s}$ with vertical and horizontal singular arcs. The spin leaves the horizontal singular arc before the point $S$ (where the control saturates the constraint) producing a bridge to reach the vertical singular line.

\section{The Numerical Methods}

In this section we present the numerical methods relating to the resolution of the contrast problem. Each method presented hereunder is a part of the package НАмРАтн and all the computations of derivatives are automatically done by the software. The geometrical study of the contrast problem ensures that the optimal solution is a concatenation of bang-singular (BS) sequences. The strategy presented in the following part is first to regularize the problem in order to detect the BS-sequences and find a good approximation of the solution. This approximation obtained by smooth continuation technique is then used to initialize the multipleshooting computation of the BS-sequences. Up to this point, the transfer duration $t_{f}$ is fixed to $1.1 t_{\mathrm{min}}$, where $t_{\min }$ is the minimal time to steer the first spin from the north pole to the center of the Bloch ball. Finally, we study the behavior of the solutions regarding the parameter $t_{f}$ and we check the second-order optimality condition by a rank test to detect any conjugate points. The methods implemented in the software HАмРАтн are developed more in details in Ref. 11 as well as the results presented in the last section.

\subsection{Homotopy and simple shooting in the smooth case}

In order to find the structure and a good approximation of the solutions of the contrast problem, we define a Tychonov ${ }^{18}$ type regularization of the problem and two such regularizations are analyzed, using continuation techniques. 
The first one will be denoted by $L^{2-\lambda}$-regularization transforms the Mayer problem in the Bolza form with cost:

$$
c\left(x\left(t_{f}\right)\right)+(1-\lambda) \int_{0}^{t_{f}}|u|^{2-\lambda} \mathrm{d} t, \quad \lambda \in[0,1],
$$

while the second one called $L^{2}$-regularization is:

$$
c\left(x\left(t_{f}\right)\right)+(1-\lambda) \int_{0}^{t_{f}}|u|^{2} \mathrm{~d} t, \quad \lambda \in[0,1] .
$$

If the control is saturating, then its value is given by $u(t)=2 \pi \cdot \operatorname{sgn}\left(H_{G}\right)$ in both cases. Otherwise, according to the maximization condition we have for the $L^{2-\lambda_{-}}$ regularization:

$$
u(x, p, \lambda)=\operatorname{sgn}\left(H_{G}\right)\left(\frac{2\left|H_{G}\right|}{(2-\lambda)(1-\lambda)}\right)^{\frac{1}{1-\lambda}},
$$

while for the $L^{2}$-regularization, the control $u$ is equal to

$$
u(x, p, \lambda)=\frac{H_{G}}{(1-\lambda)},
$$

where $p(\cdot)$ is the adjoint vector resulting from the Pontryagin Maximum Principle. Substituting $u(x, p, \lambda)$ into the Hamiltonians describing both regularizations, we have the following Hamiltonian system:

$$
\frac{\mathrm{d} x}{\mathrm{~d} t}=\frac{\partial H}{\partial p}(x, p, \lambda), \quad \frac{\mathrm{d} p}{\mathrm{~d} t}=-\frac{\partial H}{\partial x}(x, p, \lambda),
$$

with

$$
H(x, p, \lambda)=p^{0}(1-\lambda)|u(x, p, \lambda)|^{2-\lambda}+H_{F}(x, p)+u(x, p, \lambda) H_{G}(x, p)
$$

or

$$
H(x, p, \lambda)=p^{0}(1-\lambda)|u(x, p, \lambda)|^{2}+H_{F}(x, p)+u(x, p, \lambda) H_{G}(x, p),
$$

which do not depend on $u$ anymore. We can now introduce the one-parameter family of shooting equations (or homotopic function), which is defined by the boundary conditions (3.8):

$$
\begin{aligned}
S: \Omega \subset \mathbb{R}^{n} \times[0,1) & \rightarrow \mathbb{R}^{n} \\
\left(\begin{array}{c}
p_{0} \\
\lambda
\end{array}\right) & \mapsto S\left(p_{0}, \lambda\right)=\left(\begin{array}{c}
x_{1}\left(t_{f}, x_{0}, p_{0}, \lambda\right) \\
x_{2}\left(t_{f}, x_{0}, p_{0}, \lambda\right)-p_{2}\left(t_{f}, x_{0}, p_{0}, \lambda\right)
\end{array}\right),
\end{aligned}
$$

with $x_{0}=x(0)=N=((0,1),(0,1))$.

For each regularization, we first solve the problem in $\lambda=0$ using a simple shooting method. We use the fortran hybrid Newton method hybrj (from the minpack library) to solve the nonlinear equation $S\left(p_{0}, 0\right)=0$. The Jacobian of the restriction 
of $S$ on $\lambda=0$ is given to hybrj and must be invertible. Note that for $\lambda=0$, both regularizations represent the same problem and so the solutions are similar.

If the interior $\Omega \subset \mathbb{R}^{n} \times(0,1)$ of the domain $\Omega$ is made only of regular points of $S$ and if the restriction of $S$ on $\lambda=0$ is a submersion, i.e.

$$
\begin{aligned}
\operatorname{rank} S^{\prime}\left(p_{0}, \lambda\right) & =n, \quad\left(p_{0}, \lambda\right) \in \AA, \\
\operatorname{rank} \frac{\partial S}{\partial p_{0}}\left(p_{0}, \lambda\right)_{\mid \lambda=0} & =n, \quad p_{0} \in \mathbb{R}^{n},
\end{aligned}
$$

then as a consequence, the level set $\{S=0\}$ is a one-dimensional submanifold of $\mathbb{R}^{n+1}$ called the path of zeros, starting from $\lambda=0$ to $\lambda=\lambda_{f}<1$. For any $r=\left(p_{0}, \lambda\right) \in \Omega, \operatorname{dim} \operatorname{ker} S^{\prime}(r)=1$ so one can define the (tangent) vector $T(r)$ as being the unique - up to orientation - unit vector in the kernel. The orientation is chosen so that the nonvanishing determinant

$$
\operatorname{det}\left[\begin{array}{c}
S^{\prime}(r) \\
{ }^{t} T(r)
\end{array}\right]
$$

has constant sign on each connected component of $\Omega$. This provides a parametrization by arclength of the connected components of $\{S=0\}$ which are computed by integrating the following differential equation $\left(\right.$ with $\left.^{\prime}=d / d s\right)$ :

$$
r^{\prime}(s)=T(r(s)), \quad r(0)=r_{0} \in\{S=0\},
$$

with $r_{0}=\left(p_{0}, 0\right)$ obtained by a first shooting. Unlike well-known predictioncorrection methods, see Ref. 1, we merely follow the path of zeros by integrating the differential system with a high order Runge-Kutta scheme, without any correction. The solution at $\lambda_{f}$ should be an approximation of the solution of the contrast problem, in order to identify the structure of the control and to initialize the multiple shooting method described in the next part.

The main issue here is to accurately compute the Jacobian of the homotopic function. In this case we have $S$ of the form $S\left(p_{0}, \lambda\right)=b\left(z\left(t_{f}, x_{0}, p_{0}, \lambda\right)\right)=b\left(z_{f}\right)$, with $b: \mathbb{R}^{2 n} \rightarrow \mathbb{R}^{n}$ and $z=(x, p)$. The derivative $b^{\prime}(z)$ is computed in the HAMPATH code by Automatic Differentiation. The derivative $\partial_{p_{0}} z\left(t_{f}, x_{0}, p_{0}, \lambda\right)$ is the solution at $t_{f}$ of the Jacobi equation:

$$
\delta \dot{z}=\frac{\partial \mathbf{H}}{\partial z}(z(t), \lambda) \delta z,
$$

with the initial value $\delta z(0)=\left(0, I_{n}\right) \in \mathbb{R}^{2 n \times n}$. In a same way, the partial derivative with respect to the homotopic parameter $\lambda, \partial_{\lambda} z\left(t_{f}, x_{0}, p_{0}, \lambda\right)$, is the solution at $t_{f}$ of:

$$
\delta \dot{z}=\frac{\partial \mathbf{H}}{\partial z}(z(t), \lambda) \delta z+\frac{\partial \mathbf{H}}{\partial \lambda}(z(t), \lambda),
$$


with the initial value $\delta z(0)=0 \in \mathbb{R}^{2 n}$. Again HamPATH uses automatic differentiation to compute $\mathbf{H}, \partial_{z} \mathbf{H}$ and $\partial_{\lambda} \mathbf{H}$.

\subsection{Multiple shooting and homotopy in the bang-singular case}

Assume to simplify that the optimal solution of the contrast problem (i.e. at $\lambda=1$ ) is made of a single BS-sequence and let us denote by $t_{1}, 0<t_{1}<t_{f}$, the switching time between the regular arc and the singular arc. By construction, $z(t), t \in\left[0, t_{f}\right]$ is a concatenation of the integral curve of $\mathbf{H}$ joining $z(0)$ and $z_{1}=z\left(t_{1}\right)$, where $u(t)=2 \pi \operatorname{sgn} H_{G}(z(t))$ and of the integral curve of $\mathbf{H}_{s}$ starting at $z_{1}$, where $z_{1}$ must satisfy Eq. (3.6). Let us denote by $\tilde{z}\left(\cdot, z_{1}, t_{1}\right), \tilde{z}=(\tilde{x}, \tilde{p})$ the singular extremal starting at $z\left(t_{1}\right)=z_{1}$.

We can define the function which maps $\left(p_{0}, t_{1}, z_{1}\right)$ to the boundary conditions, to Eq. (3.6) and to the matching conditions $z\left(t_{1}, x_{0}, p_{0}\right)=z_{1}$. This leads to the multiple shooting function, see Refs. 8 and 17:

$$
\begin{aligned}
S: \mathbb{R}^{3 n+1} & \rightarrow \mathbb{R}^{3 n+1} \\
& \left(\begin{array}{c}
p_{0} \\
t_{1} \\
z_{1}
\end{array}\right) \mapsto\left(\begin{array}{c}
H_{G}\left(z_{1}\right) \\
\dot{H}_{G}\left(z_{1}\right) \\
z\left(t_{1}, x_{0}, p_{0}\right)-z_{1} \\
\tilde{x}_{1}\left(t_{f}, z_{1}, t_{1}\right) \\
\left\langle\tilde{x}_{2}\left(t_{f}\right) \mid \tilde{p}_{2}\left(t_{f}\right)\right\rangle-\left|\tilde{x}_{2}\left(t_{f}\right)\right|^{2}
\end{array}\right),
\end{aligned}
$$

which is of the form $S\left(p_{0}, t_{1}, z_{1}\right)=b\left(t_{1}, z_{1}, z\left(t_{1}, x_{0}, p_{0}\right), \tilde{z}\left(t_{f}, z_{1}, t_{1}\right)\right)$. We again use a Newton type algorithm to solve the nonlinear equation $S\left(p_{0}, t_{1}, z_{1}\right)=0$. We need the Jacobian of $S$, which is quite different from the smooth case. The partial derivatives of $b$ are more intricate than before and are computed again using Automatic Differentiation. As in the smooth case, $\partial_{p_{0}} z\left(t_{1}, x_{0}, p_{0}\right)$ and $\partial_{z_{1}} \tilde{z}\left(t_{f}, z_{1}, t_{1}\right)$ are solutions of the Jacobi equations (4.1), respectively with the Hamiltonian vector fields $\mathbf{H}$ and $\mathbf{H}_{s}$. Then $\partial_{t_{1}} z\left(t_{1}, x_{0}, p_{0}\right)$ is simply equal to $\mathbf{H}\left(z\left(t_{1}, x_{0}, p_{0}\right)\right)$ and finally $\partial_{t_{1}} \tilde{z}\left(t_{f}, z_{1}, t_{1}\right)$ is the solution at $t_{f}$ of:

$$
\delta \dot{z}=\frac{\partial \mathbf{H}_{s}}{\partial z}\left(z\left(t, z_{1}, t_{1}\right)\right) \delta z,
$$

with the initial value $\delta z\left(t_{1}\right)=-\mathbf{H}_{s}\left(z_{1}\right) \in \mathbb{R}^{2 n}$.

Up to this point, the final time $t_{f}$ was fixed. One important problem is to study the behavior of the solutions regarding the parameter $t_{f}$. This is done by changing the parametrization of our problem so that our new time $\bar{t}$ is in $[0,1]$. One can introduce $t_{f} \cdot \bar{t}=t$ which leads to consider $t_{f}$ as a homotopic parameter. The Hamiltonians are multiplied by $t_{f}$ and we have a one-parameter family of multiple shooting equations of the form $S\left(p_{0}, \bar{t}_{1}, z_{1}, t_{f}\right), \bar{t}_{1}=t_{1} / t_{f}$, which can be solved by continuation techniques presented in Sec. 4.1. 


\subsection{Second-order sufficient optimality conditions}

The second order sufficient conditions of optimality are related to the concept of conjugate point. According to Definition 3.2, a time $t_{c} \in\left(t_{1}, t_{f}\right]$, is a conjugate time if there exists a Jacobi field $\delta z(\cdot)=(\delta q(\cdot), \delta p(\cdot))$, semi-vertical at $t_{1}$ and $t_{c}$. Hence, a Jacobi field along a singular extremal, semi-vertical at $t_{1}$ must satisfy these three conditions:

(i)

$$
\begin{aligned}
\mathrm{d} H_{G}\left(z\left(t_{1}\right)\right) \cdot \delta z\left(t_{1}\right)= & -\delta z_{1} \cdot p_{y_{1}}+\delta y_{1} \cdot p_{z_{1}}-\delta z_{2} \cdot p_{y_{2}}+\delta y_{2} \cdot p_{z_{2}} \\
& -z_{1} \cdot \delta p_{y_{1}}+y_{1} \cdot \delta p_{z_{1}}-z_{2} \cdot \delta p_{y_{2}}+y_{2} \cdot \delta p_{z_{2}} \\
= & 0 \\
\mathrm{~d}\left\{H_{F}, H_{G}\right\}\left(z\left(t_{1}\right)\right) \cdot \delta z\left(t_{1}\right)= & -\delta_{1} \cdot \delta z_{1} \cdot p_{y_{1}}-\delta_{1} \cdot \delta y_{1} \cdot p_{z_{1}}-\delta_{2} \cdot \delta z_{2} \cdot p_{y_{2}} \\
& -\delta_{2} \cdot \delta y_{2} \cdot p_{z_{2}}+\left(\gamma_{1}-\delta_{1} \cdot z_{1}\right) \cdot \delta p_{y_{1}}-\delta_{1} \cdot y_{1} \cdot \delta p_{z_{1}} \\
& +\left(\gamma_{2}-\delta_{2} \cdot z_{2}\right) \cdot \delta p_{y_{2}}-\delta_{2} \cdot y_{2} \cdot \delta p_{z_{2}} \\
= & 0 ;
\end{aligned}
$$

(ii) $\delta x\left(t_{1}\right) \in \operatorname{vect}\left\{G\left(x\left(t_{1}\right)\right)\right\}$;

(iii) $p\left(t_{1}\right) \delta p\left(t_{1}\right)=0$ (linearization of $\left|p\left(t_{1}\right)\right|=1$ which breaks the homogeneity in $\left.\left(p^{0}, p\right)\right)$, which means $\left[0_{1 \times n}{ }^{t} p\left(t_{1}\right)\right] \cdot \delta z\left(t_{1}\right)=0$.

The second condition can be rewritten as follows. Since $G\left(x\left(t_{1}\right)\right)$ is nonzero, there exists $A \in L\left(\mathbb{R}^{n}, \mathbb{R}^{n-1}\right)$ of maximal rank $(n-1)$ such as vect $\left\{G\left(x\left(t_{1}\right)\right)\right\}=\operatorname{ker} A$ $\left(A \in \mathbb{R}^{n-1 \times n}\right.$ is formed by the vectors of a basis of $\left.\left\{G\left(x\left(t_{1}\right)\right)\right\}^{\perp}\right)$; let us denote $B=\left[A 0_{n-1 \times n}\right] \in L\left(\mathbb{R}^{2 n}, \mathbb{R}^{n-1}\right)$, then $\delta x\left(t_{1}\right) \in \operatorname{vect}\left\{G\left(x\left(t_{1}\right)\right)\right\}$ is equivalent to $B \cdot \delta z\left(t_{1}\right)=0$.

Finally if we denote by $C$ the matrix:

$$
C=\left(\begin{array}{c}
0_{1 \times n}{ }^{t} p\left(t_{1}\right) \\
d H_{G}\left(z\left(t_{1}\right)\right) \\
d\left\{H_{F}, H_{G}\right\}\left(z\left(t_{1}\right)\right) \\
B
\end{array}\right) \in \mathbb{R}^{(n+2) \times 2 n},
$$

then all the three conditions are $C \cdot \delta z\left(t_{1}\right)=0$. Under our assumptions $C$ is of maximal rank, then it has a kernel of dimension $2 n-(n+2)=n-2$, which defines two Jacobi fields $\delta z_{i}(t)=\left(\delta x_{i}(t), \delta p_{i}(t)\right), i=1,2$, if $n=4$.

A time $t_{c}>t_{1}$ is a conjugate time if we have the following rank condition:

$$
\operatorname{rank}\left\{\delta x_{1}\left(z\left(t_{c}\right)\right), \delta x_{2}\left(z\left(t_{c}\right)\right), G\left(x\left(t_{c}\right)\right)\right\}<3 .
$$

Using assumption (A3), this is equivalent to:

$$
\operatorname{det}\left\{\delta x_{1}\left(z\left(t_{c}\right)\right), \delta x_{2}\left(z\left(t_{c}\right)\right), G\left(x\left(t_{c}\right)\right), F\left(x\left(t_{c}\right)\right)\right\}=0 .
$$




\section{Numerical Results in the Case of Blood Samples}

\subsection{Comparison of the two homotopies}

When $\lambda$ is equal to zero, the two homotopies coincide; remarkably, both are initialized by using a trivial guess for the unknown initial adjoint state, $p_{0}=$ 5 e $-2 \times(1,1,1,1)$. The solution Fig. 3 for $\lambda=0$ is obtained with an error of $10^{-14}$ on the norm of the shooting function. The path of zeros is then followed up to some $\lambda_{f}<1$, revealing the BS structure of the control (see Figs. 4 and 5 ). The evolution of the contrast along the path is given Fig. 6. The path itself is represented in Fig. 7. All results of this subsection and the following are obtained for $t_{f}$ fixed to 1.1 times the minimum time of the single spin system with the same boundary conditions as spin No. 1.

\subsection{Resolution of the contrast problem}

The value obtained at $\lambda_{f}<1$ for each homotopy is a candidate as initial guess for the multiple shooting when one assumes the BS structure. One also needs to
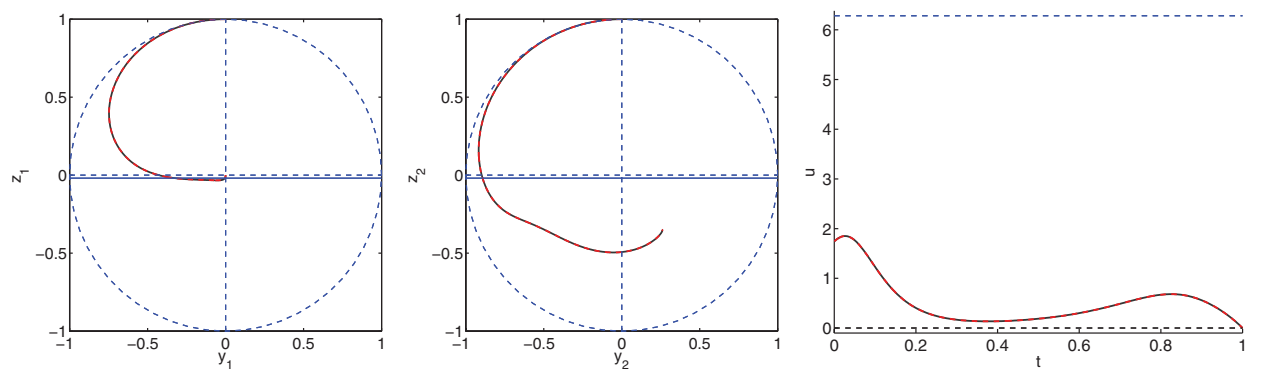

Fig. 3. (Color online) Solution for $\lambda=0\left(t_{f}=1.1 \times \min t_{f}\right)$. Trajectories for spin 1 and 2 in the $(y, z)$-plane are portrayed in the first two subgraphs. In each subgraph, the solution associated with the $L^{2}$-homotopy (resp. $L^{2-\lambda}$ ) is the dashed red line (resp. black line). The corresponding controls are drawn in the rightmost subgraph. For $\lambda=0$, the two homotopies define the same problem, so trajectories and controls are identical for both.
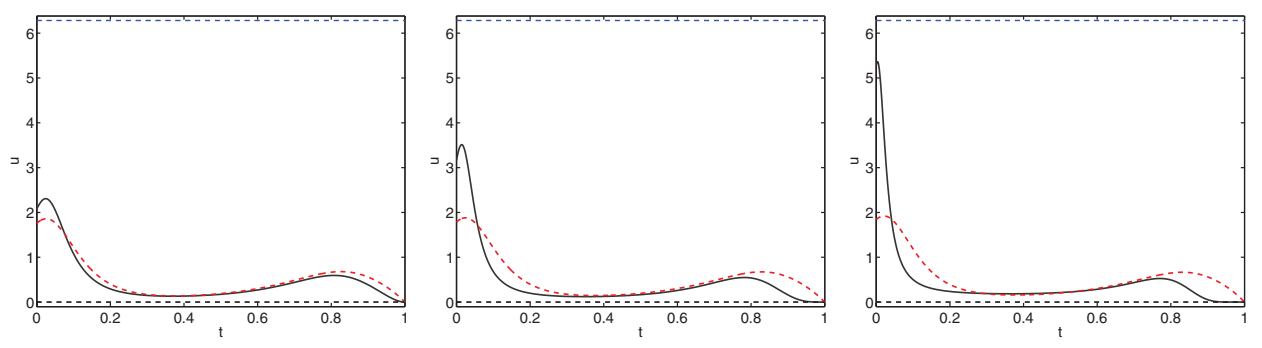

Fig. 4. (Color online) Controls for $\lambda=0.5,0.8,0.9\left(t_{f}=1.1 \times \min t_{f}\right)$. In each subgraph, the solution associated with the $L^{2}$-homotopy (resp. $L^{2-\lambda}$ ) is the dashed red line (resp. black line). As $\lambda \rightarrow 1$, the BS structure is numerically revealed for by the $L^{2-\lambda}$-homotopy. It is less visible for the $L^{2}$-homotopy. 

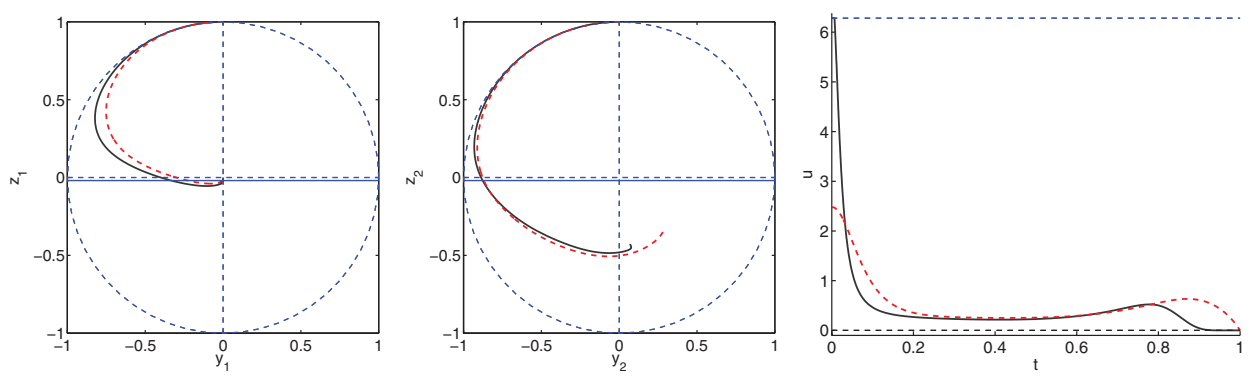

Fig. 5. (Color online) Solutions for $\lambda_{f}<1\left(t_{f}=1.1 \times \min t_{f}\right)$. Trajectories and controls as in Fig. 3. In the $L^{2}$ case, $\lambda_{f}=0.99$; in the $L^{2-\lambda}$ case, $\lambda_{f}=0.92$.

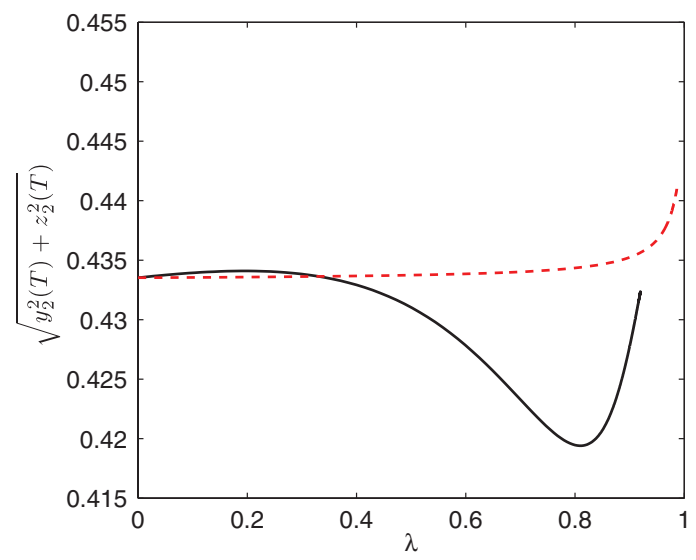

Fig. 6. (Color online) Contrast along the path $\left(t_{f}=1.1 \times \min t_{f}\right)$. The contrast at the final state is evaluated for each homotopy as $\lambda$ ranges $\left[0, \lambda_{f}\right]$. The value associated with the $L^{2}$-homotopy (resp. $L^{2-\lambda}$ ) is the dashed red line (resp. black line). Although it is not clear whether the contrasts associated with each of the two homotopies converges to the same value as $\lambda \rightarrow 1$ (see also Fig. 7), the corresponding solutions allow to initialize the resolution for $\lambda=1$ and lead then to the same solutions of the contrast problem (see Sec. 5.2).

initialize the unknown junction time $t_{1}$ between the bang and singular arc. A whole range of initializations is considered as illustrated by Fig. 8 . These various initializations lead to three different solutions (see Figs. 9-11), no matter the homotopy chosen to initialize $p_{0}$. The influence of the initial guess for $t_{1}$ on the result is discussed Fig. 12. The error on the norm of the shooting function is at most equal to $10^{-10}$ for any solution.

\subsection{Influence of the final time}

Given that the initial point is a stationary point, the constrast is an increasing function of $t_{f}$ acting as a parameter. Indeed, applying a zero control at $t=0$ leaves the system in its initial state so there is an inclusion of admissible controls between problems when the final time is increased (and the bigger the set of controls, the 

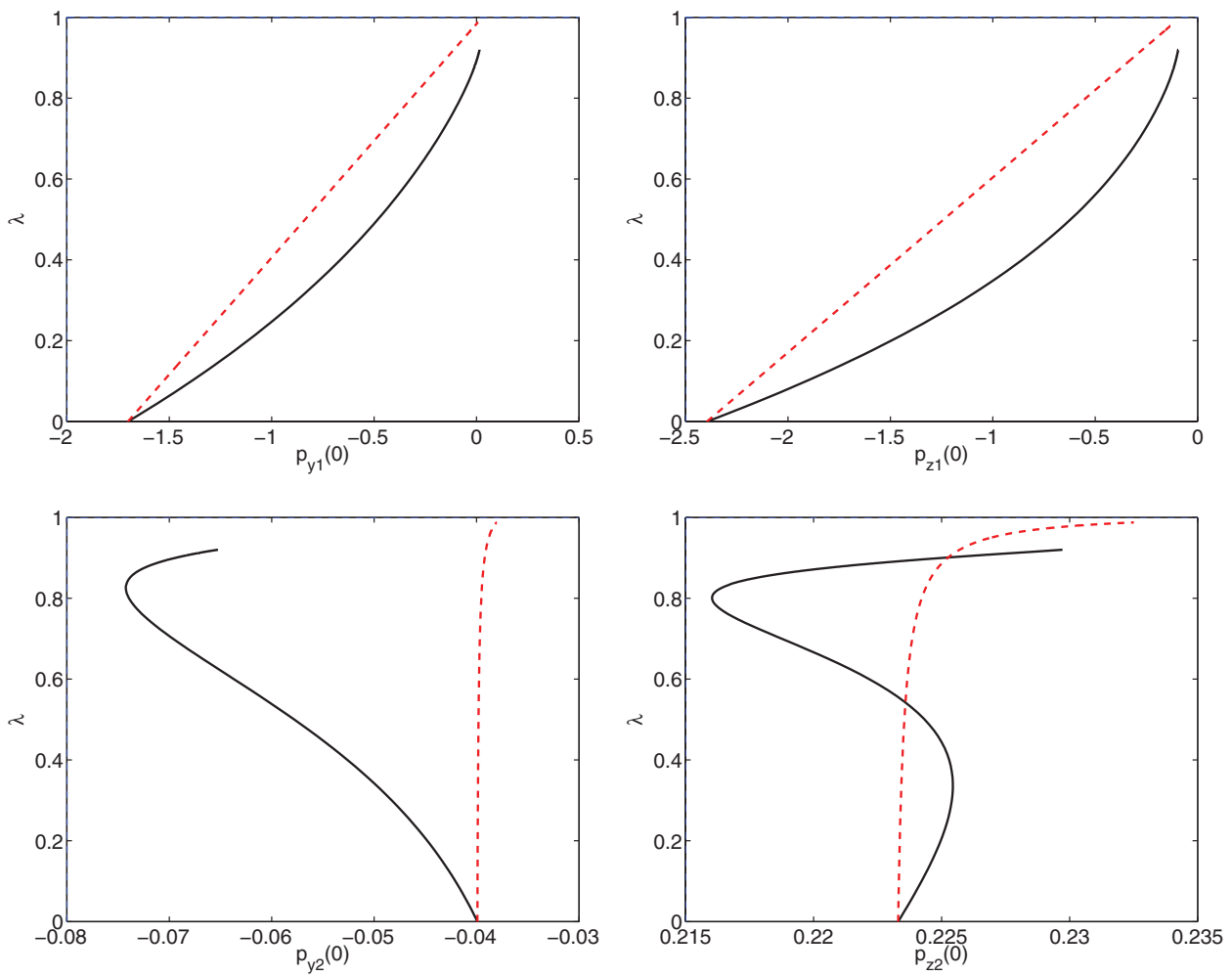

Fig. 7. (Color online) Path of zeros $\left(t_{f}=1.1 \times \min t_{f}\right)$. For each component of the unknown initial costate, the path associated with the $L^{2}$-homotopy (resp. $L^{2-\lambda}$ ) is the dashed red line (resp. black line). The main differences when $\lambda \rightarrow 1$ are observed on $p_{y_{2}}$ and $p_{z_{2}}$.

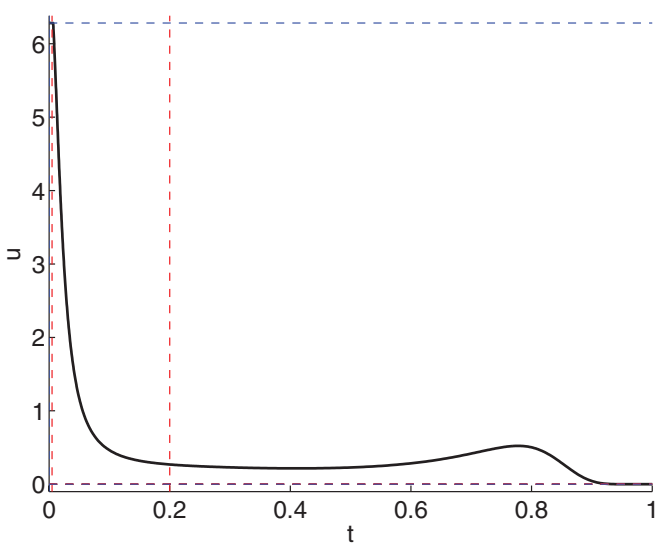

Fig. 8. (Color online) Discretization of the time interval to define initial guesses for $t_{1}\left(t_{f}=\right.$ $\left.1.1 \times \min t_{f}\right)$. The initializations of the unknown junction time $t_{1}$ from the bang to the singular arc are chosen in the subinterval $[0.005,0.2]$. This heuristic choice is related to the structure of the control for $\lambda_{f}$ (here, the control obtained for the $L^{2-\lambda}$ homotopy is portrayed). The corresponding initialization for $z_{1}=z\left(t_{1}\right)$ is obtained by merely integrating the dynamics for $\lambda_{f}$. 

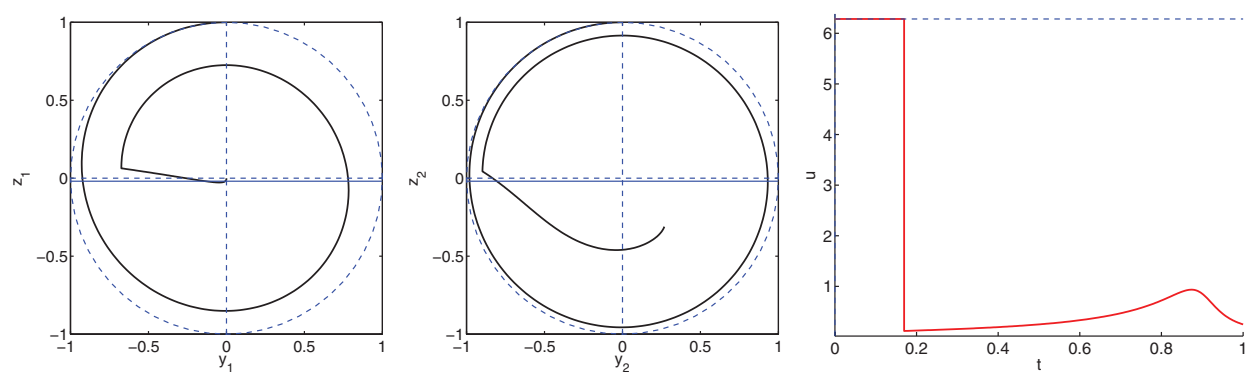

Fig. 9. (Color online) Contrast problem, solution No. 1 with contrast $0.411\left(t_{f}=1.1 \times \min t_{f}\right)$. Trajectories for spin 1 and 2 in the $(y, z)$-plane are portrayed in the first two subgraphs. The corresponding BS control is drawn in the rightmost subgraph.
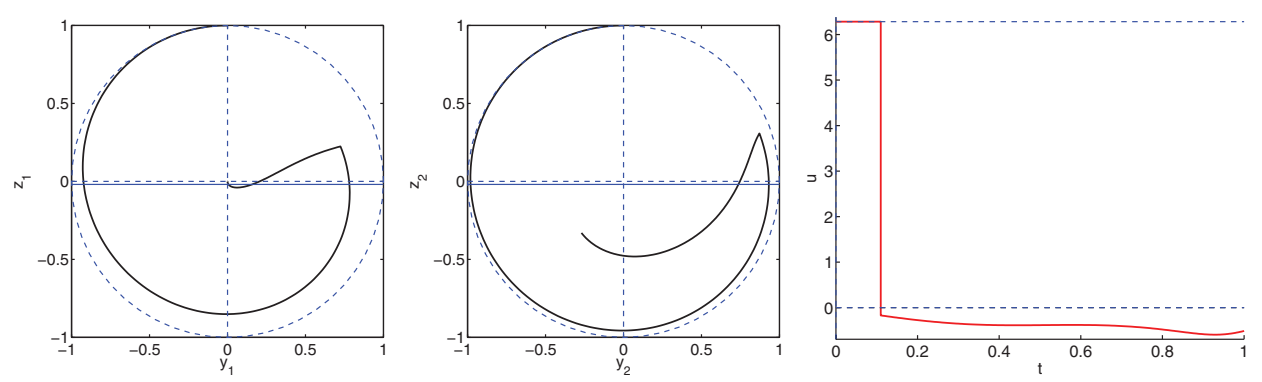

Fig. 10. (Color online) Contrast problem, solution No. 2 with contrast $0.426\left(t_{f}=1.1 \times \min t_{f}\right)$. Trajectories for spin 1 and 2 in the $(y, z)$-plane are portrayed in the first two subgraphs. The corresponding BS control is drawn in the rightmost subgraph.
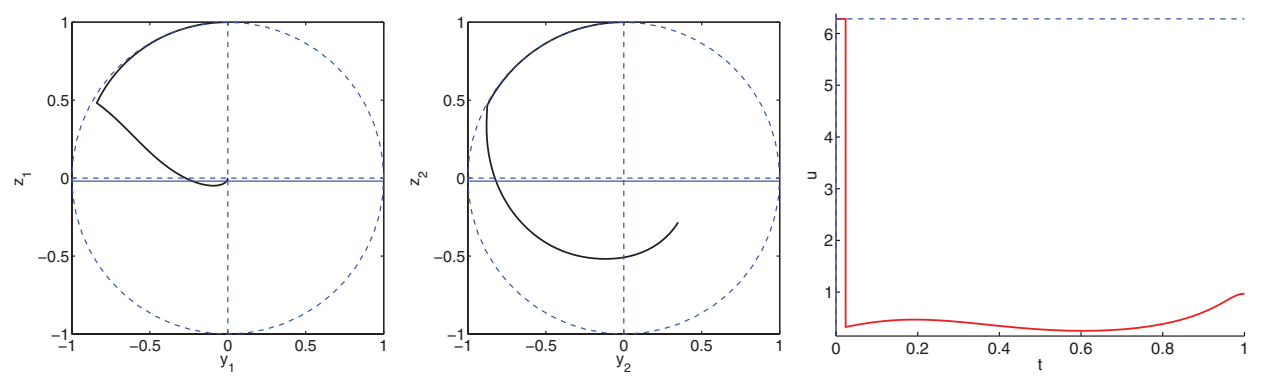

Fig. 11. (Color online) Contrast problem, solution No. 3 with contrast $0.449\left(t_{f}=1.1 \times \min t_{f}\right)$. Trajectories for spin 1 and 2 in the $(y, z)$-plane are portrayed in the first two subgraphs. The corresponding BS control is drawn in the rightmost subgraph.

larger the maximum contrast). Whereas the branch Nos. 1 and 2 generated by the solutions for $t_{f}=1.1 \times \min t_{f}$ provide local but not global minima, it is not possible to decide whether branch 3 corresponds to global minimizers. Accordingly, even branch 3 only provides a lower bound for the value function (see Fig. 13). Having increasing bounded (by one, which is the maximum possible contrast given the final condition on spin No. 1) functions, it is natural to expect asymptotes 

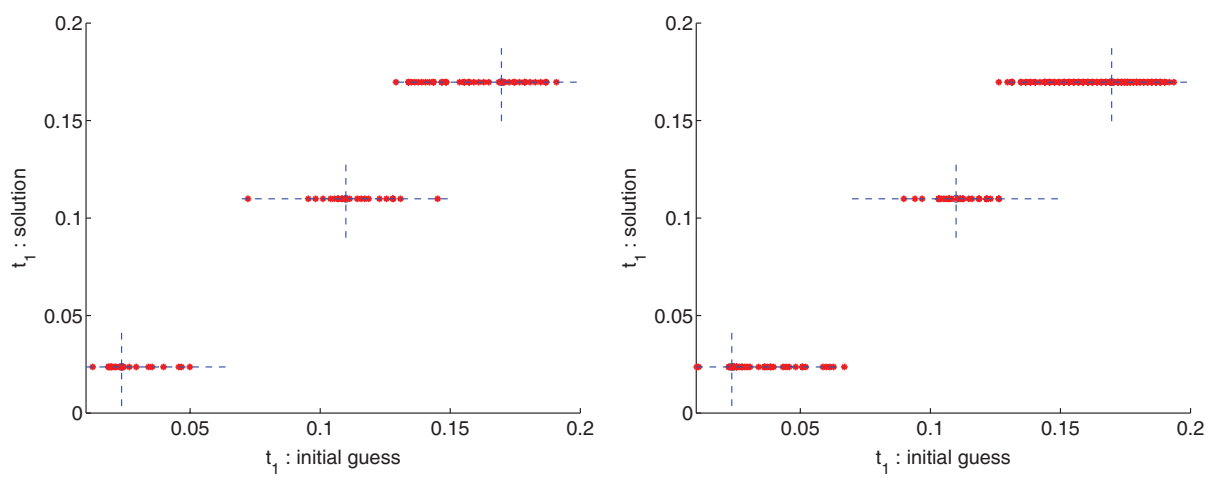

Fig. 12. (Color online) Influence of the initial guess for $t_{1}$ on the contrast solution $\left(t_{f}=1.1 \times\right.$ $\left.\min t_{f}\right)$. For each value of the initial guess on the $x$-axis, the solution for $t_{1}$ is plotted when convergence is obtained. The initial costate $p_{0}$ is either initialized by the solution for $\lambda_{f}$ of the $L^{2-\lambda}$-homotopy (L.H.S.), or the $L^{2}$-homotopy (R.H.S.). It is clear from Figs. 9, 10, 11, that the smaller $t_{1}$, the better the contrast: Solution Nos. $1,2,3$ are obtained for $t_{1}=0.17,0.11$ and 0.024 , respectively.
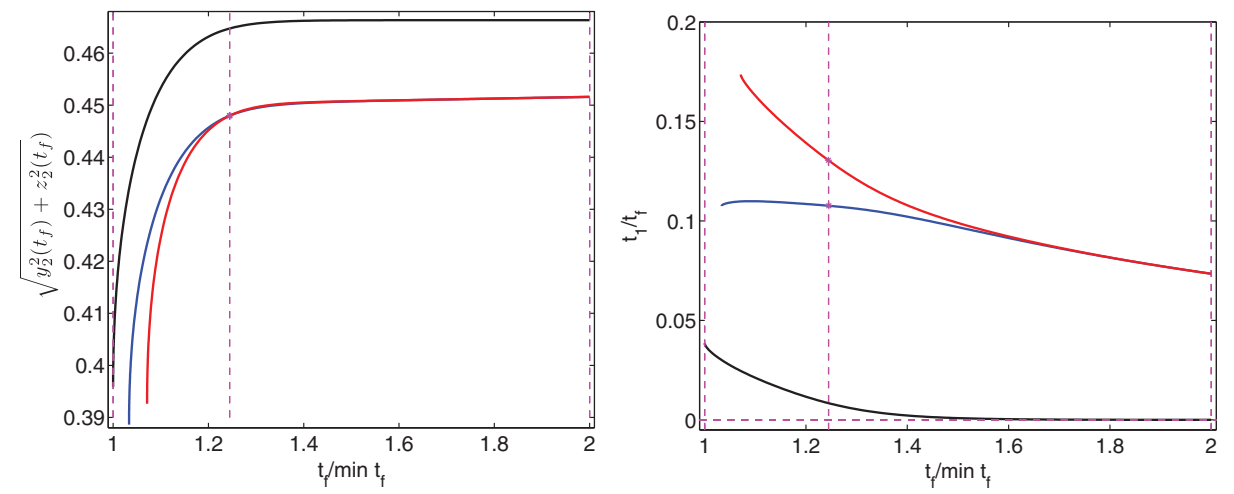

Fig. 13. (Color online) Evolution of the contrast with respect to the final time. On the left, the contrast increases as $t_{f}$ is varied up to two times the minimum time of the one spin system for solution No. 1 (red line), 2 (blue) and 3 (black line). For $t_{f}>1.3 \times \min t_{f}$, the asymptotic value on each branch of solutions is almost reached (up to $99.5 \%$ of the maximal contrast). Moreover, although the costs associated with branch Nos. 1 and 2 are indiscernible past such final times, the strategies still differ as illustrated on the rightmost subgraph (see also Figs. 18 and 19). At $1.244 \times \min t_{f}$, the costs cross and then tend to the same value for larger $t_{f}$. On the right, the evolution of the junction time $t_{1}$ is portrayed for each branch of solution. On one hand, for $t_{f} \simeq 1.244 \times \min t_{f}$, the values of $t_{1}$ still differ between branch Nos. 1 and 2 . This difference tends to vanish for larger $t_{f}$. On the other hand, $t_{1} \rightarrow 0$ on branch 3 as $t_{f}$ grows, entailing that the bang arc tends to disappear.

on each branch. The numerical simulations indicate that the limit value on the three branches is almost reached past $t_{f} \simeq 1.3 \times \min t_{f}$. For large enough final times, solutions 1 and 2 coincide for symmetry reasons (see Figs. 18-19) while the bang arc tends to disappear on solution 3 (see Fig. 20). Conversely, a BSBS 


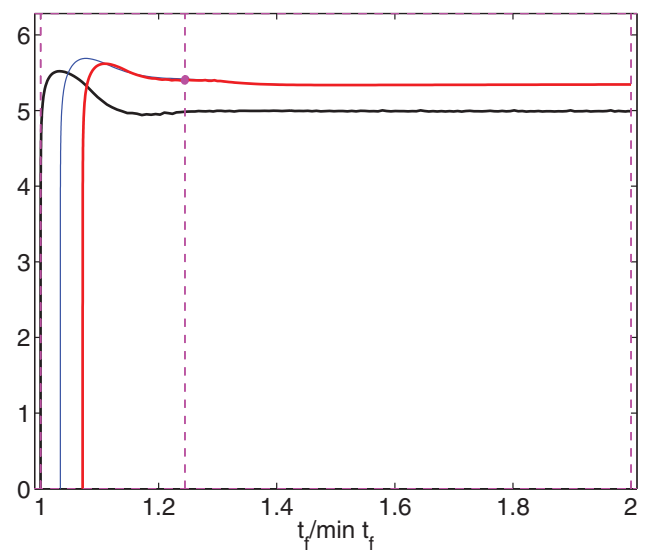

Fig. 14. (Color online) The minimal distance between the singular control along the trajectory and the upper bound $2 \pi$ with respect to the normalized final time, for solution No. 1 (red line), 2 (blue) and 3 (black line). The distance tends to 0 when $t_{f}$ decreases. When it is 0 , the singular control is saturating and another bang arc is required. For solutions Nos. 1 and 2, the distance is 0 for $t_{f}$ greater than $\min t_{f}$. On the other hand, for solution No. 3 the distance tends to 0 as $t_{f}$ tends to $\min t_{f}$ and the BSBS-sequence of the single spin case is revealed (see Fig. 18).
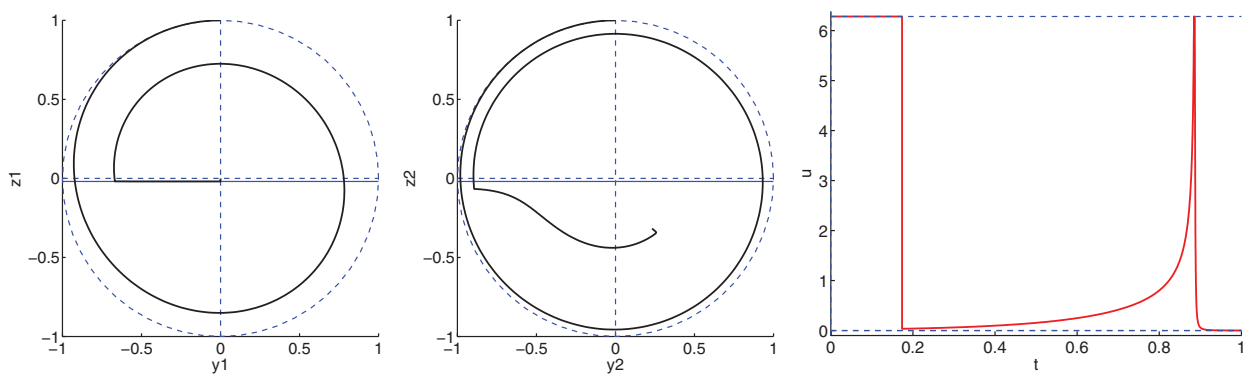

Fig. 15. (Color online) Solution No. $1\left(t_{f}=1.07 \times \min t_{f}\right)$. Trajectories for spin 1 and 2 in the $(y, z)$-plane are portrayed in the first two subgraphs for solution No. 1. The corresponding control is drawn in the rightmost subgraph. As $t_{f} \rightarrow \min t_{f}$, the BSBS structure is revealed as the singular subarc comes closer to saturating the constraint $|u| \leq 2 \pi$.
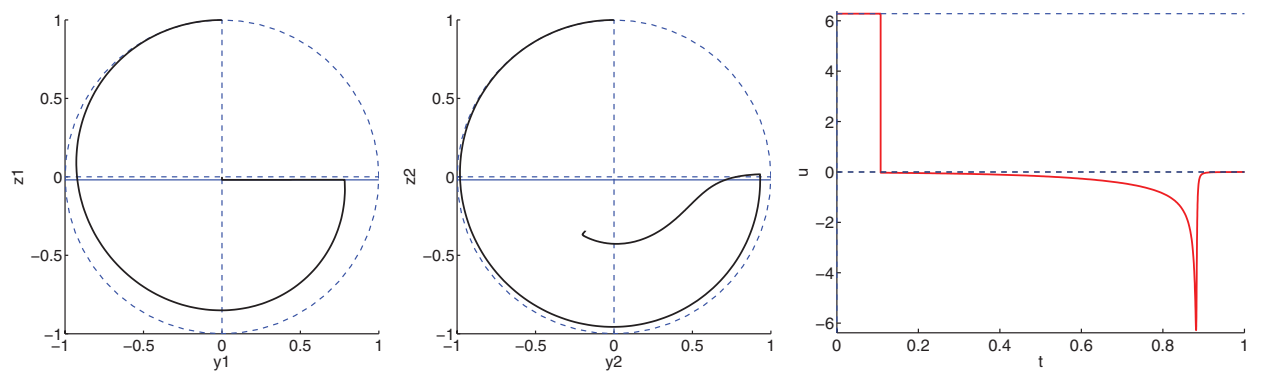

Fig. 16. (Color online) Solution No. $2\left(t_{f}=1.03 \times \min t_{f}\right)$. Same as Fig. 15 . 

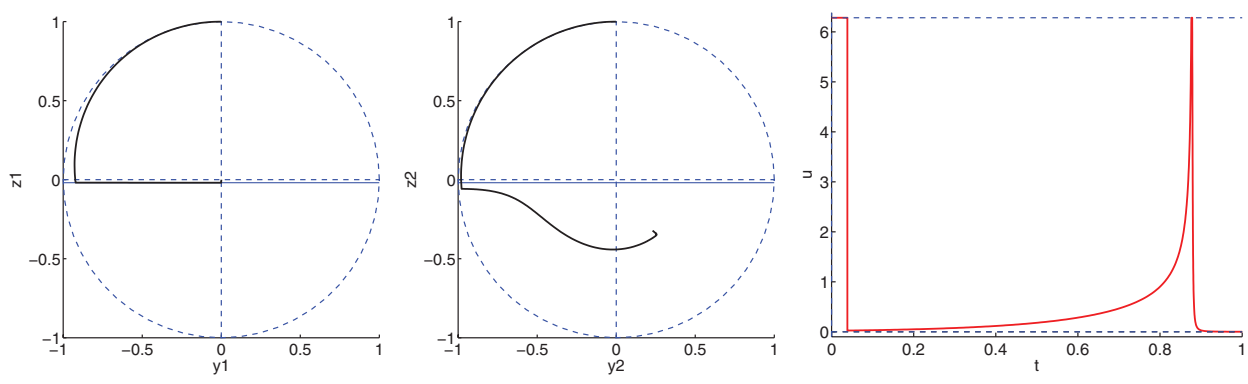

Fig. 17. (Color online) Solution No. $3\left(t_{f}=1.000004 \times \min t_{f}\right)$. Same as Fig. 15.
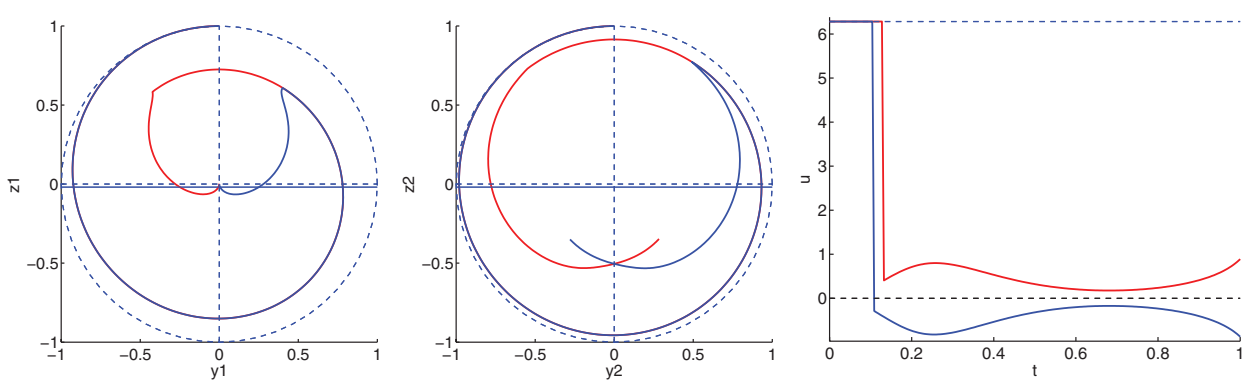

Fig. 18. (Color online) Solution Nos. 1 and $2\left(t_{f}=1.244 \times \min t_{f}\right)$. Trajectories for spin 1 and 2 in the $(y, z)$-plane are portrayed in the first two subgraphs for solution No. 1 (red line) and 2 (blue line). The corresponding controls are drawn in the rightmost subgraph. Despite the small difference between the durations of the bang arcs, the difference in strategies is clear and associated with a symmetry of the system.
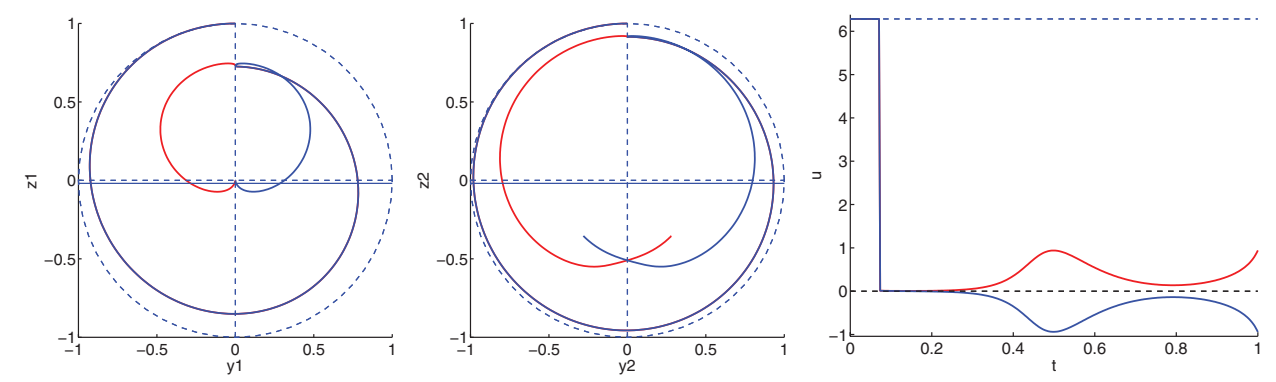

Fig. 19. (Color online) Solution Nos. 1 and $2\left(t_{f}=2 \times \min t_{f}\right)$. Trajectories for spin 1 and 2 in the $(y, z)$-plane are portrayed in the first two subgraphs for solution No. 1 (red line) and 2 (blue line). The corresponding controls are drawn in the rightmost subgraph. The durations of the bang arcs are equal and the singular controls are opposite. The singular trajectories are symmetrical with respect to the $z$-axis. 

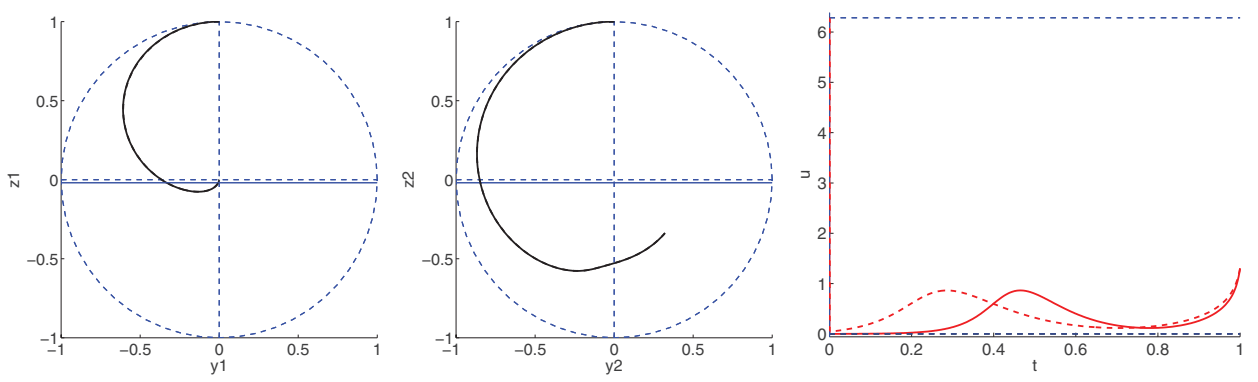

Fig. 20. (Color online) Solution No. 3: $t_{f}=1.5 \times \min t_{f}$ (dashed line) and $t_{f}=2 \times \min t_{f}$ (filled line). Trajectories for spin 1 and 2 in the $(y, z)$-plane are portrayed in the first two subgraphs for solution No. 3. The corresponding control is drawn in the rightmost subgraph. The trajectories are superimposed but not the controls. The bang arc tends to disappear and the singular control makes the particles resting at the initial point close to the north pole during more time.
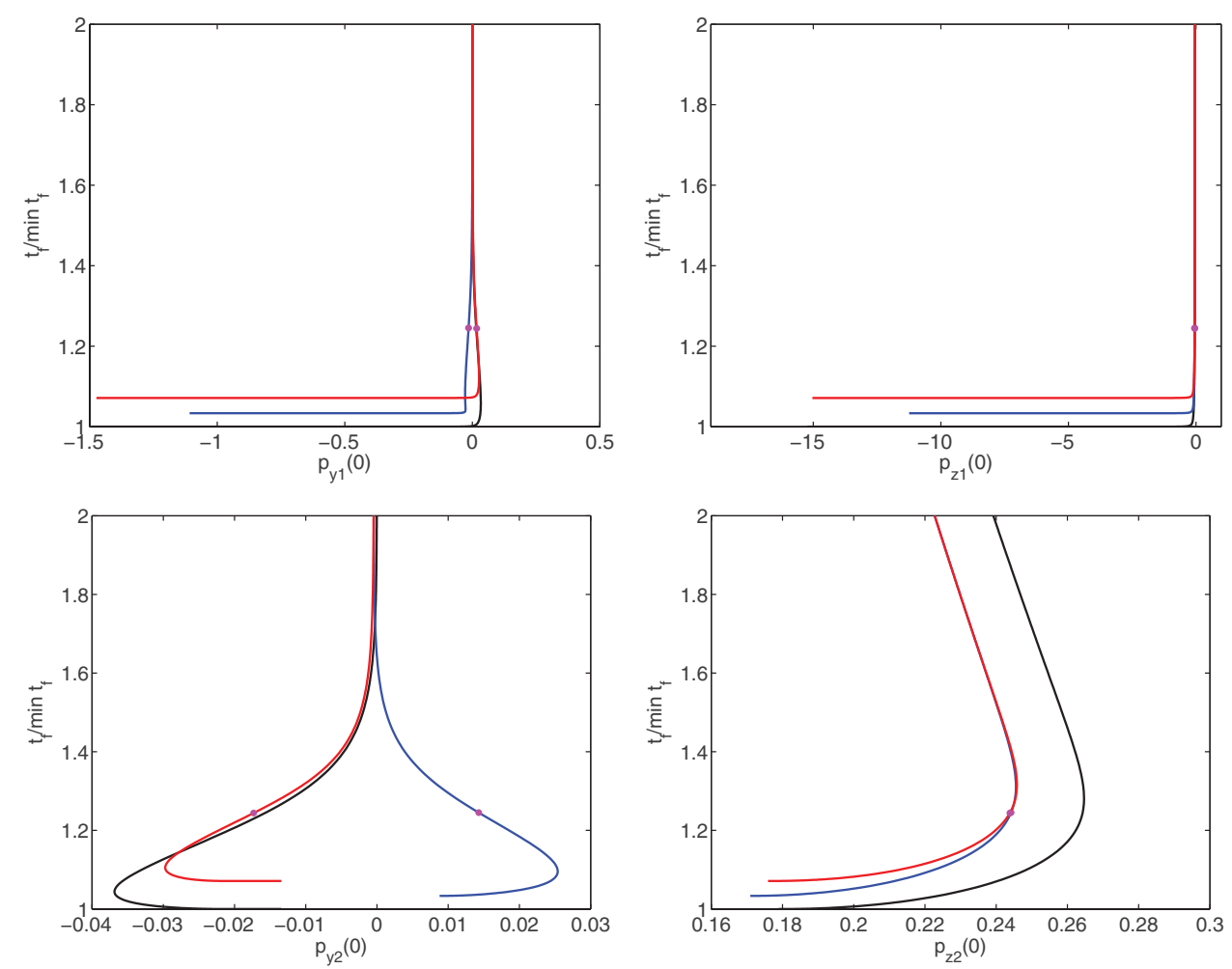

Fig. 21. (Color online) Path of zeros for the continuation on the final time. For each component of the unknown initial costate, branches associated with solution No. 1 (red line), 2 (blue line) and 3 (black line) are portrayed. The convergence of branches 1 and 2 is observed as $t_{f}$ increases, in coherence with the convergence on $t_{1}$ detailed Fig. 13. The only persisting difference with branch No. 3 comes from the $p_{y_{2}}$ and $p_{z_{2}}$ components of the initial adjoint state. 
structure seems to be revealed on the third branch as $t_{f} \rightarrow \min t_{f}$ (see Figs. 14-17). The corresponding three paths of zeros are represented on Fig. 21. Second order condition checks are also performed along branch 3 as illustrated Fig. 22, ensuring local optimality by virtue of Theorem 3.2.
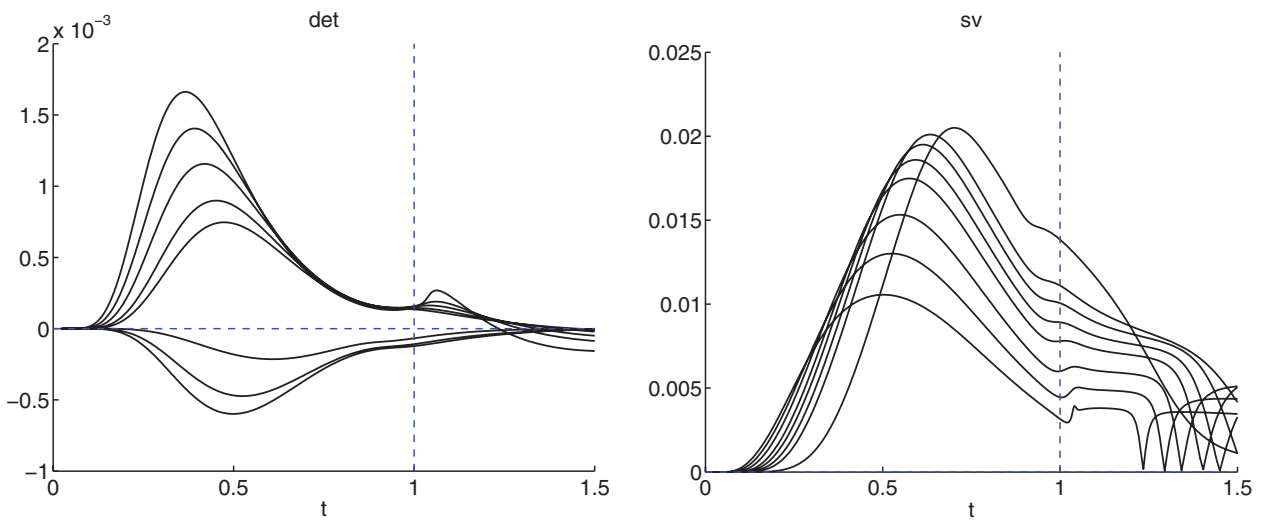

Fig. 22. Second order sufficient condition check on branch No. 3. The rank condition resulting from Theorem 3.2 is evaluated for several values of the ratio $t_{f} / \min t_{f}$ in $(1,1.15]$ for the branch of solutions No. 3 that provide the best contrast. On the left, for each $t_{f}$, the curve is reparametrized so that the final time corresponds to the abscissa 1; the determinant associated with the rank condition is plotted, so there is a conjugate time whenever it vanishes. One observes that conjugate times on each extremal are located after the (normalized to 1) final time, ensuring local optimality of the trajectory. On the right, the determinant test is confirmed using a singular value decomposition on the associated matrix. The smallest singular value is plotted.
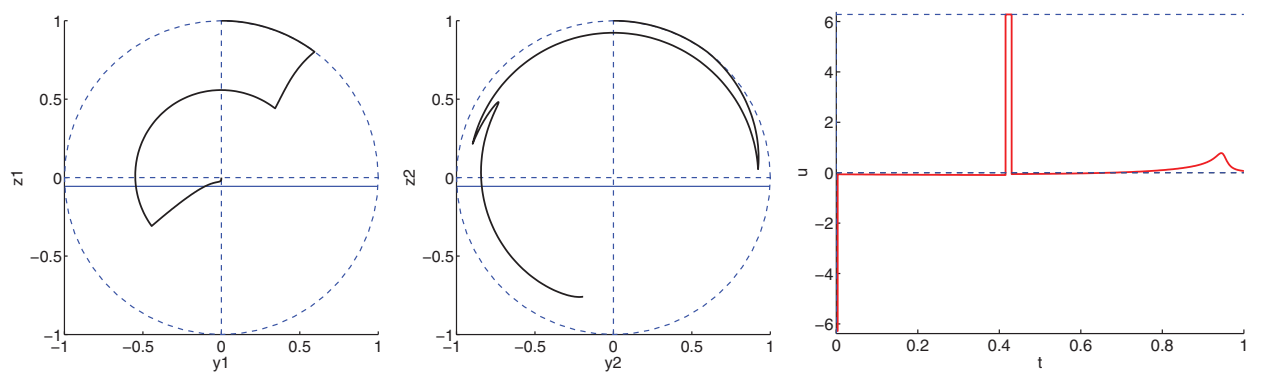

Fig. 23. (Color online) Contrast problem in the fluid/water case, solution BSBS with contrast 0.783 for a transfer time $t_{f}=1.5 \times \min t_{f}$. Trajectories for spin 1 (i.e. cerebrospinal fluid with $T_{1}=2000 \mathrm{~ms}$ and $T_{2}=200 \mathrm{~ms}$ ) and spin 2 (i.e. water with $T_{1}=2500 \mathrm{~ms}$ and $T_{2}=2500 \mathrm{~ms}$ ) in the $(y, z)$-plane are portrayed in the first two subgraphs. The corresponding control is drawn in the rightmost subgraph. 

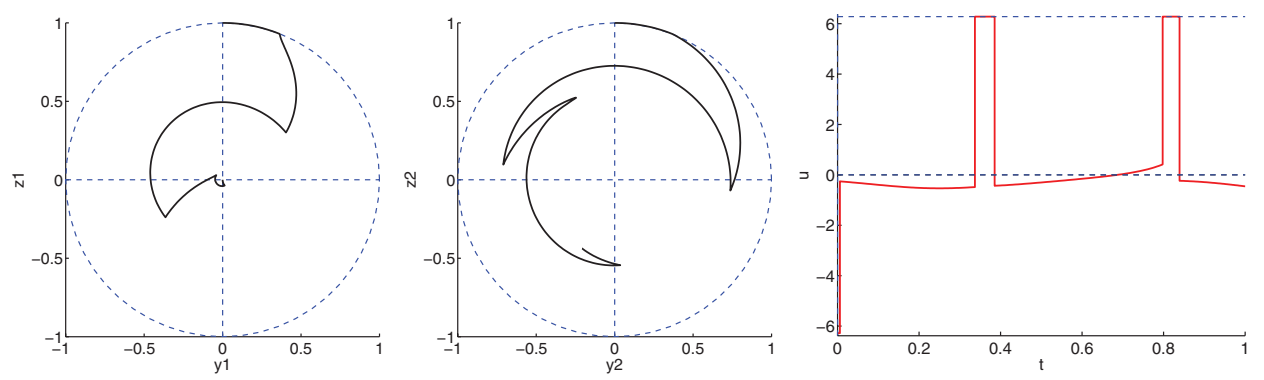

Fig. 24. (Color online) Contrast problem in the blood case, solution BSBSBS with contrast 0.484 for a transfer time $t_{f}=1.5 \times \min t_{f}$, from continuation from the fluid/water case. Trajectories for spin 1 (i.e. deoxygenated blood with $T_{1}=1350 \mathrm{~ms}$ and $T_{2}=50 \mathrm{~ms}$ ) and spin 2 (i.e. oxygenated blood $T_{1}=1350 \mathrm{~ms}$ and $\left.T_{2}=200 \mathrm{~ms}\right)$ in the $(y, z)$-plane are portrayed in the first two subgraphs. The corresponding control is drawn in the rightmost subgraph.

\section{Conclusion}

In this paper, we have presented the geometric optimal methods combined with numerical adapted schemes implemented in the HAMPATH code which lead to calculate the optimal control in the NMR-imaging techniques for the blood sample case. Restricting to a single control field, a contrast of 0.466 was obtained for a BSsequence and a transfer time of $1.5 \times \min t_{f}$, where $\min t_{f}$ is the minimum time to drive the first spin to zero magnetization. This gives a local optimum with respect to all solutions contained in a tubular neighborhood of the singular arc.

The same techniques can be applied to other cases, e.g. cerebrospinal fluid/water samples. In the analysis presented in this paper, the continuation method is applied to regularize the Mayer problem to Bolza and a second continuation concerns the transfer time. Similarly a continuation method can be made on the relaxation parameters to study the different cases.

As a conclusion, to illustrate the extension of the approach we present a numerical result. A BSBS-sequence, see Fig. 23, is computed thanks to the regularization process in the cerebrospinal fluid/water case and a continuation is made towards the blood case which leads to a change of the structure in BSBSBS, see Fig. 24, and provides a contrast of 0.484 for the $3 \mathrm{BS}$-sequence for a transfer time of $1.5 \times \min t_{f}$ versus 0.466 for a BS-sequence of the blood case analyzed in this article.

\section{Acknowledgments}

B.B. was supported by PEPS INSIS optimal control of spin dynamics in NMR and ANR Programme Blanc GCM, Project No. NT09_504490. O.C. was supported by Conseil Régional de Bourgogne, Contract No. 2009-160E-160-CE-160T.

\section{References}

1. E. Allgower and K. Georg, Introduction to Numerical Continuation Methods, Classics in Applied Mathematics Vol. 45 (SIAM, 2003). 
2. V. G. Boltyanskiur, R. V. Gamkrelidze, E. F. Mishchenko and L. S. Pontryagin, The Mathematical Theory of Optimal Processes (Gordon \& Breach, 1986).

3. B. Bonnard and M. Chyba, Singular Trajectories and Their Role in Control Theory, Mathematics \& Applications (Springer-Verlag, 2003).

4. B. Bonnard, O. Cots, S. Glaser, M. Lapert, D. Sugny and Y. Zhang, Geometric optimal control of the contrast imaging problem in nuclear magnetic resonance, IEEE Trans. Automat. Control 57 (2012) 1957-1969.

5. B. Bonnard and I. Kupka, Théorie des singularités de l'application entrée/sortie et optimalité des trajectoires singulières dans le problème du temps minimal, Forum Math. 5 (1993) 111-159.

6. M. Braun, S. J. Glaser, M. Lapert, D. Sugny and Y. Zhang, Singular extremals for the time-optimal control of dissipative spin 1/2 particles, Phys. Rev. Lett. 104 (2010), pp. 083001.

7. R. Brockett, S. J. Glaser and N. Khaneja, Sub-Riemannian geometry and time optimal control of three spin systems: Quantum gates and coherence transfer, Phys. Rev. A 65 (2002) 11.

8. R. Bulirsch and J. Stoer, Introduction to Numerical Analysis, 2nd edn., Texts in Applied Mathematics, Vol. 12 (Springer-Verlag, 1993).

9. J.-B. Caillau, O. Cots and J. Gergaud, Намpath: On solving optimal control problems by indirect and path following methods, apo.enseeiht.fr/hampath (2010).

10. J.-B. Caillau, O. Cots and J. Gergaud, Differential continuation for regular optimal control problems, Optim. Methods Soft. 27 (2011) 177-196.

11. O. Cots, Contrôle optimal géométrique: Méthodes homotopiques et applications, Ph.D. thesis, http://cots.perso.math.cnrs.fr, Institut Mathématiques de Bourgogne, Dijon, France, 2012.

12. S. V. Fomin and I. M. Gelfand, Calculus of Variations, revised English edn. translated and edited by Richard A. Silverman (Prentice-Hall, 1963).

13. A. J. Krener, The high order maximal principle and its application to singular extremals, SIAM J. Control Optim. 15 (1977) 256-293.

14. I. Kupka, Geometric theory of extremals in optimal control problems. The fold and Maxwell case, Trans. Amer. Math. Soc. 299 (1987) 225-243.

15. M. Lapert, Développement de nouvelles techniques de contrôle optimal en dynamique quantique: de la RMN à la Physique moléculaire. Ph.D. thesis, Université de Bourgogne, Dijon, France, 2011.

16. M. Lapert, Y. Zhang, M. A. Janich, S. J. Glaser and D. Sugny, Exploring the physical limits of saturation contrast in magnetic resonance imaging, Sci. Rep. 2 (2012) 589.

17. H. Maurer, Numerical solution of singular control problems using multiple shooting techniques, J. Optim. Theory Appl. 18 (1976) 235-257.

18. N. Moïsséev, Problèmes Mathématiques d'analyse Des Systèmes. Traduit du Russe: Mathématiques. [Translations of Russian Works: Mathematics] (Mir, 1985).

19. H. G. Moyer, Sufficient conditions for a strong minimum in singular control problems, SIAM J. Control 11 (1973) 620-636. 